\title{
A single bacterial genus maintains root development in a complex microbiome
}

Omri M. Finkel ${ }^{1,2 \dagger}$, Isai Salas-González ${ }^{1,2,3 \dagger}$, Gabriel Castrillo1,2†‡, Jonathan M. Conway ${ }^{1,2 \dagger}$, Theresa F. Law ${ }^{1,2}$, Paulo José Pereira Lima Teixeira' ${ }^{1,20}$, Ellie D. Wilson ${ }^{1,2}$, Connor R. Fitzpatrick ${ }^{1,2}$, Corbin D. Jones ${ }^{1,3,4,5,6,7}$, Jeffery L. Dangl $1^{1,2,3,6,7,8^{*}}$

'Department of Biology, University of North Carolina at Chapel Hill, Chapel Hill, North Carolina, United States of America.

${ }^{2}$ Howard Hughes Medical Institute, University of North Carolina at Chapel Hill, Chapel Hill, North Carolina, United States of America.

${ }^{3}$ Curriculum in Bioinformatics and Computational Biology, University of North Carolina at Chapel Hill, Chapel Hill, North Carolina, United States of America.

${ }^{4}$ Department of Genetics, University of North Carolina at Chapel Hill, Chapel Hill, North Carolina, United States of America.

${ }^{5}$ Lineberger Comprehensive Cancer Center, University of North Carolina at Chapel Hill, Chapel Hill, North Carolina, United States of America.

${ }^{6}$ Carolina Center for Genome Sciences, University of North Carolina at Chapel Hill, Chapel Hill, North Carolina, United States of America.

${ }^{7}$ Curriculum in Genetics and Molecular Biology, University of North Carolina at Chapel Hill, Chapel Hill, North Carolina, United States of America.

${ }^{8}$ Department of Microbiology and Immunology, University of North Carolina at Chapel Hill, Chapel Hill, North Carolina, United States of America.

*Correspondence to: dangl@email.unc.edu

†These authors contributed equally to this work.

$\ddagger$ Current address: Future Food Beacon of Excellence and the School of Biosciences, University of Nottingham, Sutton Bonington, United Kingdom

aCurrent address: Department of Biology, "Luiz de Queiroz" College of Agriculture (ESALQ), University of São Paulo (USP), Piracicaba, São Paulo, Brazil 


\section{Abstract}

2 Plants grow within a complex web of species interacting with each other and with the

3 plant. Many of these interactions are governed by a wide repertoire of chemical signals,

4 and the resulting chemical landscape of the rhizosphere can strongly affect root health

5 and development. To understand how microbe-microbe interactions influence root

6 development in Arabidopsis, we established a model system for plant-microbe-microbe-

7 environment interactions. We inoculated seedlings with a 185-member bacterial

8 synthetic community (SynCom), manipulated the abiotic environment, and measured

9 bacterial colonization of the plant. This enabled classification of the SynCom into four

10 modules of co-occurring strains. We deconstructed the SynCom based on these

11 modules, identifying microbe-microbe interactions that determine root phenotypes.

12 These interactions primarily involve a single bacterial genus, Variovorax, which

13 completely reverts severe root growth inhibition (RGl) induced by a wide diversity of

14 bacterial strains as well as by the entire 185-member community. We demonstrate that

15 Variovorax manipulate plant hormone levels to balance this ecologically realistic root community's effects on root development. We identify a novel auxin degradation operon

17 in the Variovorax genome that is necessary and sufficient for RGI reversion. Therefore, 18 metabolic signal interference shapes bacteria-plant communication networks and is 19 essential for maintaining the root's developmental program. Optimizing the feedbacks 20 that shape chemical interaction networks in the rhizosphere provides a promising new 21 ecological strategy towards the development of more resilient and productive crops. 


\section{Main}

23 Plant phenotypes, and ultimately fitness, are influenced by the microbes living in close 24 association with them ${ }^{1-4}$. These microbes, collectively termed the plant microbiota, assemble based on plant- and environmentally-derived cues ${ }^{5,6}$ resulting in myriad plantmicrobe interactions. Beneficial and detrimental microbial effects on plants can be either

27 direct ${ }^{1,7-10}$, or an indirect consequence of microbe-microbe interactions ${ }^{3,11}$. The contribution of microbe-microbe interactions within the microbiota to community assembly and to the community's effect on the host is unknown, and it is therefore unclear to what extent binary plant-microbe interactions hold in complex ecological contexts. While antagonistic microbe-microbe interactions are known to play an important role in shaping plant microbiota and protecting plants from pathogens ${ }^{3}$, another potentially significant class of interactions is metabolic signal interference ${ }^{8,12}$ : rather than direct antagonism, microbes interfere with the delivery of chemical signals produced by other microbes, altering plant-microbe signaling ${ }^{13-15}$.

Plant hormones, in particular auxins, are both produced and degraded by an abundance of plant-associated microbes ${ }^{16-19}$. Microbially-derived auxins can have effects on plants ranging from growth-promoting to disease-inducing, depending on context and concentration $^{20}$. The plant's intrinsic root developmental patterns are dependent on finely calibrated auxin and ethylene concentration gradients with fine differences across

41 tissues and cell types ${ }^{21}$, and it is not known how the plant integrates exogenous,

42 microbially-derived auxin fluxes into its developmental plan.

44 Here we apply a synthetic community (SynCom) that is reasonably representative of wild 45 soil root-associated microbiomes, to axenic plants to ask how microbe-microbe 
interactions shape plant root development. We use plant colonization patterns across

4716 abiotic conditions to guide stepwise deconstruction of the SynCom, leading to the

48 identification of multiple levels of microbe-microbe interactions that interfere with the

49 additivity of bacterial effects on root development. We demonstrate that a single

50 bacterial genus, Variovorax, is required for maintaining the root's intrinsically controlled

51 developmental program by tuning its chemical landscape. Finally, we identify the locus

52 conserved across Variovorax strains that is responsible for this phenotype.

Consistent community assembly across environmental conditions

55 To model plant-microbiota interactions in a fully controlled setting, we established a plant-microbiota microcosm representing the native bacterial root-derived microbiota on

57 agar plates. We inoculated 7-day-old seedlings with a defined 185-member bacterial SynCom (Extended Data Fig. 1a, Supplementary Table 1) composed of genomesequenced isolates obtained from surface sterilized Arabidopsis roots (Methods 1). The composition of this SynCom captures the diversity of Actinobacteria, Proteobacteria and

61 Bacteroidetes; the three major root-enriched phyla ${ }^{6,22,23}$; and Firmicutes, which are 62 abundant in plant-associated culture collections ${ }^{24}$. To test the robustness of microbiota assembly to the abiotic environment, we exposed this microcosm to each of 16 different abiotic contexts by manipulating one of four variables (salinity, temperature, phosphate concentration, and $\mathrm{pH})$. We measured SynCom composition in root, shoot and agar fractions 12 days post-inoculation using 16S rRNA amplicon sequencing.

67 
plant enrichment patterns seen in soil-planted Arabidopsis thaliana, with comparable proportions of Proteobacteria, Bacteroidetes and Actinobacteria (Extended Data Fig. 1b). Firmicutes, which are not plant-enriched, were reduced to $<0.1 \%$ of the relative abundance. We observed similar patterns in seedlings grown in sterilized potting soil ${ }^{25}$ inoculated with the same SynCom (Methods 2). Both relative abundances and plant enrichment patterns at the unique sequence (USeq)-levels were significantly correlated between the agar- and soil-based systems, confirming the applicability of the relatively high-throughput agar-based system as a model for plant microbiota assembly (Extended Data Fig. 1c).

Fraction (substrate, root, shoot) (Extended Data Fig. 1d), explained most (40\%) of the variance across all abiotic variables. Abiotic conditions significantly affected both alpha(Extended Data Fig. 1e) and beta-diversity (Fig. 1a). We calculated pairwise correlations in relative abundance across all samples, and identified four well-defined modules of cooccurring strains: A, B, C and D (Fig. 1b, Supplementary Table 2). These modules formed distinct phylogenetically-structured guilds in association with the plant: module A contained mainly Gammaproteobacteria and was predominantly more abundant in the substrate than in the seedling; module B contained mainly low-abundance Firmicutes, with no significant seedling enrichment trend; modules $C$ and D were composed mainly 88 of Alphaproteobacteria and Actinobacteria, respectively, and showed plant-enrichment across all abiotic conditions (Figure 1a, Supplementary Table 2). Both

90 Alphaproteobacteria (module C) and Actinobacteria (module D) are consistently plant91 enriched across plant species ${ }^{23}$, suggesting that these clades contain plant-association 
92 traits that are deeply rooted in their evolutionary histories.

Root development is controlled by multiple microbe-microbe interactions

95 We next asked whether the different modules of co-occurring strains play different roles

in determining plant phenotypes (Methods 3). We inoculated seedlings with SynComs composed of modules A, B, C and D singly, or in all six possible pairwise module combinations, and imaged the seedlings 12 days post-inoculation. We observed strong primary root growth arrest in seedlings inoculated with plant-enriched modules C or D

(Fig. 2a, c). Root growth inhibition (RGl) did not occur in seedlings inoculated with modules A or B, which do not contain plant-enriched strains (Fig. 2a, Supplementary Table 3). To test whether the root phenotype derived from each module is an additive outcome of its individual constituents, we inoculated seedlings in mono-association with each of the 185 SynCom members (Methods 4). We observed that 34 taxonomically

105 diverse strains, distributed across all four modules, induced RGI (Fig. 2b, Extended Data

106 Fig. 2, Supplementary Table 4). However, neither the full SynCom nor derived SynComs

107 consisting of modules A or B exhibited RGI (Fig. 2a). Thus, binary plant-microbe interactions were not predictive of interactions in this complex community context.

In seedlings inoculated with module pairs, we observed an epistatic interaction: in the

presence of module A, RGI caused by modules $C$ and D was reverted (Fig. 2a). Thus, through deconstructing the SynCom into four modules, we found that bacterial effects on root development are governed by multiple levels of microbe-microbe interactions.

114 This is exemplified by at least four instances: within modules A or B and between module 
115 A and modules $C$ or D. Since three of these interactions involve module A, we predicted 116 that this module contains strains that strongly attenuate RGI, preserving stereotypic root

117 development.

Variovorax are necessary and sufficient to maintain stereotypic plant root development within complex microbiota

121 To identify strains within module $A$ responsible for intra- and inter-module RGI

122 attenuation, we reduced our system to a tripartite plant-microbe-microbe system

123 (Methods 5). We individually screened the 18 non-RGI strains from module A for their

124 ability to attenuate RGI caused by representative strains from all four modules. We found

125 that all strains from a single genus, Variovorax (Family Comamonadaceae), suppressed

126 RGl caused by representative RGl-inducing strains from module C (Agrobacterium

127 MF224) and module D (Arthrobacter CL28; Fig. 2d, Supplementary Table 5). The strains

128 from modules A (Pseudomonas MF48) and B (Bacillus MF107), were not suppressed by

129 Variovorax, but rather by two closely related Burkholderia strains (CL11, MF384). A

130 similar pattern was observed when we screened three selected RGl-suppressing

131 Variovorax strains (CL14, MF160) and Burkholderia CL11, against a diverse set of RGI-

132 inducers. Variovorax attenuated 13 of the 18 RGl-inducers tested (Fig. 2e, 133 Supplementary Table 5).

135 To test whether RGI induction and repression observed on agar occur in soil as well, we 136 germinated Arabidopsis on sterile soil inoculated with an RGI-suppressor-inducer pair 137 composed of the RGI-inducer Arthrobacter CL28 and the RGl-attenuator Variovorax 
CL14 (Methods 2). As expected, Arthrobacter CL28 induced RGI which was reverted by Variovorax CL14 in soil (Fig. 2f, Supplementary Table 6). We generalized this observation by showing that Variovorax-mediated RGI attenuation extended to tomato seedlings, where Variovorax CL14 reverted Arthrobacter CL28-mediated RGI (Extended Data Fig. 3a, Supplementary Table 7, Methods 6). Finally, we tested whether the RGI-suppressor strains maintain their capacity to attenuate RGI in the context of the full 185 -member community (Methods 7). We compared the root phenotype of seedlings exposed to either the full SynCom or to the same community dropping-out all ten Variovorax strains and/or all six Burkholderia strains present in this SynCom (drop-out system, Fig. 3a, b, c). We found that Variovorax are necessary and sufficient to revert RGI within the full community (Fig. 3b, c, Supplementary Table 8). Further, the presence of Variovorax increases the plant's total root network length (Extended Data Fig. 3b). This result was robust across a range of substrates, including soil, and under various biotic and abiotic contexts (Fig. 3d, e, f, Extended Data Fig. 3b, 4, Supplementary Table 8; Methods 8, 9).

To ascertain the phylogenetic breadth of the Variovorax ability to attenuate RGI, we tested additional Variovorax strains from across the genus' phylogeny (Extended Data Fig. 5, Supplementary Table 1; Methods 10). All 19 tested Variovorax reverted RGI induced by Arthrobacter CL28 (Methods 11). A strain from the nearest plant-associated outgroup to this genus, Acidovorax Root21926, did not revert RGI (Extended Data Fig. 5, Supplementary Table 9). Thus, all tested strains representing the broad phylogeny of Variovorax interact with a wide diversity of bacteria to enforce stereotypic root development within complex communities, independent of biotic or abiotic contexts. 
162 We tested whether Variovorax attenuate RGI by inhibiting growth of RGl-inducing strains

163 (Methods 12). We counted colony forming units of the RGI inducer Arthrobacter CL28

164 from roots in the presence or absence of Variovorax CL14 and found that CL28

165 abundance increased in the presence of Variovorax CL14 (Extended Data Fig. 6, 166 Supplementary Table 10). To test whether Variovorax modulates bacterial abundances

167 in the whole community, we compared the bacterial relative abundance profiles in 168 seedlings colonized with the full SynCom to that colonized with the Variovorax drop-out 169 community (Methods 8d). We found no changes in the abundances of RGl-inducing 170 strains in response to the Variovorax drop-out (Fig. 3g). Notably, Variovorax account for

171 only $\sim 1.5 \%$ of the root community (Fig. $3 \mathrm{~h}$ ). These results rule out the possibility that

172 Variovorax enforce stereotypic root developmentby antagonizing or outcompeting RGI-

173 inducers.

Variovorax maintain root development through auxin and ethylene manipulation

176 To study the mechanisms underlying bacterial effects on root development, we analysed

177 the transcriptomes of seedlings colonized for 12 days with the RGl-inducing strain

178 Arthrobacter CL28 and the RGl-attenuator strain Variovorax CL14, either in mono179 association with the seedling or in a tripartite combination (Fig. 2f; Methods 13). We also 180 performed RNA-Seq on seedlings colonized with the full SynCom (no RGI) or the 181 Variovorax drop-out SynCom (RGl; Fig. 3a). Eighteen genes were significantly induced 182 only under RGI conditions across both experiments (Fig. 4a, b, Supplementary Table 11). 183 Seventeen of these are co-expressed genes with proposed functions related to the root 
$184 \operatorname{apex}^{27}$ (Fig 4b, Extended Data Fig. 7). The remaining gene is indole-3-acetic acid-amido synthetase GH3.2, which conjugates excess amounts of the plant hormone auxin and is a robust marker for late auxin responses ${ }^{28,29}$ (Fig. 4b). Production of auxins is a welldocumented mechanism by which bacteria modulate plant root development ${ }^{13}$. Indeed, the top 12 auxin-responsive genes from an RNA-Seq study examining acute auxin response in Arabidopsis ${ }^{28}$ exhibited an average transcript increase in seedlings exposed to our RGI-inducing conditions (Fig. 4c and Supplementary Table 12). We hypothesized that RGI attenuation by Variovorax is likely mediated by interference with bacterially-

192 produced auxin signalling.

We asked whether RGI-attenuation by Variovorax is directly and exclusively related to auxin signalling (Methods 14). Besides auxin, other small molecules cause RGl. These include the plant hormones ethylene ${ }^{30}$ and cytokinin $^{31}$; and microbial-associated molecular patterns (MAMPs) including the flagellin-derived peptide flg22 32 . We tested the ability of diverse Variovorax strains and of the Burkholderia strain CL11 to revert RGI induced by auxins (Indole-3-acetic acid [IAA] and the auxin analogue 2,4Dichlorophenoxyacetic acid [2,4-D]), ethylene (the ethylene precursor 1201 Aminocyclopropane-1-carboxylic acid [ACC]), cytokinins (Zeatin, 6-Benzylaminopurine) and flg22 peptide (Fig. 4d). All tested Variovorax suppressed RGI induced by IAA or ACC

203 (Fig. 4d, Supplementary Table 13), with the exception of Variovorax YR216 which did not 
207 attenuated RGI induced by $2,4-\mathrm{D}$, by flg22 or by cytokinins, indicating that Variovorax

208 revert RGl induction by interfering with auxin and/or ethylene signalling. Furthermore,

209 this function is mediated by recognition of auxin by Variovorax and not by the plant auxin

210 response per se, since the auxin response (RGI) induced by 2,4-D is not reverted. Indeed,

211 we found that Variovorax CL14 degrades IAA in culture (Extended Data Fig. 8a; Methods

212 15) and quenches fluorescence of the Arabidopsis auxin reporter line DR5::GFP caused

213 by the RGI inducer Arthrobacter CL28 (Fig. 4e, Extended Data Fig. 8b, Supplementary

214 Table 14; Methods 17).

215

216 To ascertain the roles of auxin and ethylene perception by the plant in responding to

217 RGl-inducers, we used the auxin-insensitive axr2-1 mutant ${ }^{33}$, combined with a 218 competitive inhibitor of ethylene receptors, 1-Methylcyclopropene (1-MCP) ${ }^{34}$, (Methods 17). We inoculated wild type seedlings and the axr2-1 mutants, treated or not with 1MCP, with the RGl-inducing Arthrobacter CL28 or the Variovorax drop-out SynCom

221 (Supplementary Table 15). We observed in both cases that bacterial RGI is reduced in axr2-1 and 1-MCP-treated wild type seedlings, and is further reduced in doubly-

223 insensitive 1-MCP-treated axr2-1 seedlings, demonstrating an additive effect of auxin 224 and ethylene on bacterially-induced RGI (Fig. 4f). Thus, a complex SynCom can induce 225 severe morphological changes in root phenotypes via both auxin- and ethylenedependent pathways, and both are reverted when Variovorax are present. 
230 To identify the bacterial mechanisms involved in RGl-attenuation, we compared the

231 genomes of the 10 Variovorax strains in the SynCom to the genomes of the other 175

232 SynCom members. We identified a list of 947 orthogroups that were rare $(<5 \%$

233 prevalence) in the 175 SynCom members and core (100\% prevalence) across the 10

234 Variovorax strains. We collapsed these orthogroups into hotspots (regions of physically

235 continuous orthogroups) and focused on the 12 hotspots that contained at least 10

236 Variovorax-unique orthogroups (Extended Data Fig. 9a, Methods 18 and Supplementary

237 Table 16). Hotspot 33 contains genes with low sequence homology (average $\sim 30 \%$

238 identity) to the genes iacCDEFR of the Paraburkholderia phytofirmans PsJN IAA-

239 degradation iac operon ${ }^{19}$ but lacks iacABI which were shown to be necessary for

240 Paraburkholderia growth on IAA $^{18}$ (Fig. 5a). To test whether the hotspots we identified

241 are responsive to $\mathrm{RGI}$-inducing bacteria, we analysed the transcriptome of Variovorax

242 CL14 in monoculture and in co-culture with the RGl-inducing strain Arthrobacter CL28

243 (Methods 19). We observed extensive transcriptional reprograming of Variovorax CL14

244 when co-cultured with Arthrobacter CL28 (Supplementary Table 17). Among the 12

245 hotspots we identified, the genes in hotspot 33 were the most highly upregulated (Fig.

246 5a, Extended Data Fig. 9b, Methods 19). We thus hypothesized that hotspot 33 contains

247 an uncharacterized auxin degradation operon.

248

249 In parallel, we constructed a Variovorax CL14 genomic library in E. coli with $>12.5 \mathrm{~kb}$

250 inserts in the broad host range vector $p B B R 1-M C S 2$, and screened the resulting E. coli

251 clones for auxin degradation (Methods 20). Two clones from the approximately 3,500

252 screened degraded IAA (Fig. 5a Vector 1 and Vector 2, Fig. 5b, Methods 20b). The 
253 Variovorax CL14 genomic inserts in both of these clones contained portions of hotspot

25433 (Fig. 5a, Extended Data Fig. 9). The overlap common to these clones contained 9

255 genes, among them the weak homologs to Paraburkholedria iacCDE. To test whether

256 this genomic region is sufficient to revert RGI in the plant, we transformed a relative of

257 Variovorax, Acidovorax Root219 that does not cause or revert RGI (Extended Data Fig.

258 5), with the shorter functional insert containing Vector 2 (V2) or with an empty vector (EV).

259 We inoculated the resulting gain-of-function strain, Acidovorax Root219::V2 or the

260 control Acidovorax Root219::EV onto plants treated with IAA or inoculated with the RGI

261 strain Arthrobacter CL28. Acidovorax Root219::V2 fully reverted IAA-induced RGI (Fig.

262 5c) and partially reverted Arthrobacter CL28-induced RGI, despite colonizing roots at

263 significantly lower numbers than Variovorax CL14 (Fig.5d, Supplementary table 18).

264 Critically, we deleted hotspot 33 from Variovorax CL14 (Fig. 5a) to test whether this

265 putative operon is necessary for RGl-reversion. The resulting strain Variovorax

266 CL14 $\triangle$ HS33 did not degrade IAA in culture (Fig. 5b) and did not revert IAA-induced (Fig.

267 5c) or Arthrobacter CL28-induced (Fig. 5d) RGl. Thus, this Variovorax-specific gene

268 cluster is necessary for the RGI-suppression phenotype and auxin degradation. It is thus

269 the critical genetic locus required by Variovorax to maintain stereotypic root development

270 in the context of a phylogenetically diverse microbiome.

271

\section{Conclusions}

273 Signalling molecules and other secondary metabolites are adaptations that allow

274 microbes to survive competition for primary metabolites. Our results illuminate the

275 importance of a trophic layer of microbes that utilize these secondary metabolites for 
276 their own benefit, while potentially providing the unselected exaptation ${ }^{35}$ of interfering

277 with signalling between the bacterial microbiota and the plant host. Metabolic signal

278 interference was demonstrated in the case of quorum quenching ${ }^{15}$, degradation of

279 MAMPs $^{36}$ and, indeed, the degradation of bacterially-produced auxin, including among

280 Variovorax ${ }^{13,14,16}$. We have shown here that the metabolic homeostasis enforced by the

281 presence of Variovorax in a phylogenetically diverse, realistic synthetic community allows

282 the plant to maintain its developmental program within a chemically complex matrix.

283 Variovorax were recently found to have the rare property of improved plant-colonization

284 upon late arrival to an established community, as opposed to arriving together with the 285 other strains in the community ${ }^{37}$, suggesting that Variovorax utilize bacterially-

286 produced/induced rather than plant-derived substrates. Furthermore, via re-analysis of

287 a recent large-scale time- and spatially-resolved survey of the Arabidopsis root

288 microbiome ${ }^{38}$, we noted that two Variovorax- $16 \mathrm{~S}$ sequences were found in $\sim 85 \%$ and

$289100 \%$ of the sampled sites (among the top 10 most prevalent sequence among

290 rhizoplane samples, Extended Data Fig. 10). These ecological observations, together

291 with our results using a reductionist microcosm, reinforce the importance of Variovorax

292 as key players in bacteria-bacteria-plant communication networks required to maintain

293 stereotypic root development within a complex biochemical ecosystem. 
a
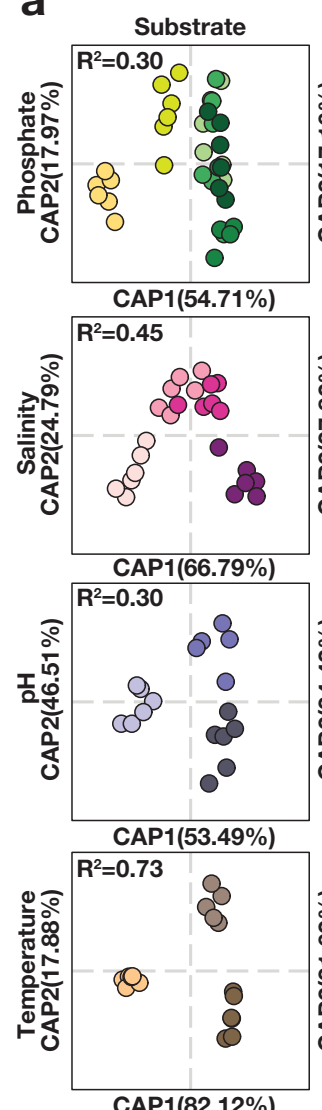

Phosphate (Panels A/B)

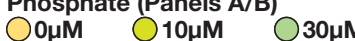
Salinity (Panels A/B)

$50 \mathrm{mM} \bigcirc 100 \mathrm{mM}$

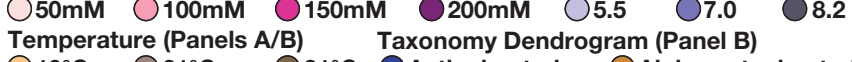
$10^{\circ} \mathrm{C} 2^{\circ} \mathrm{C}$
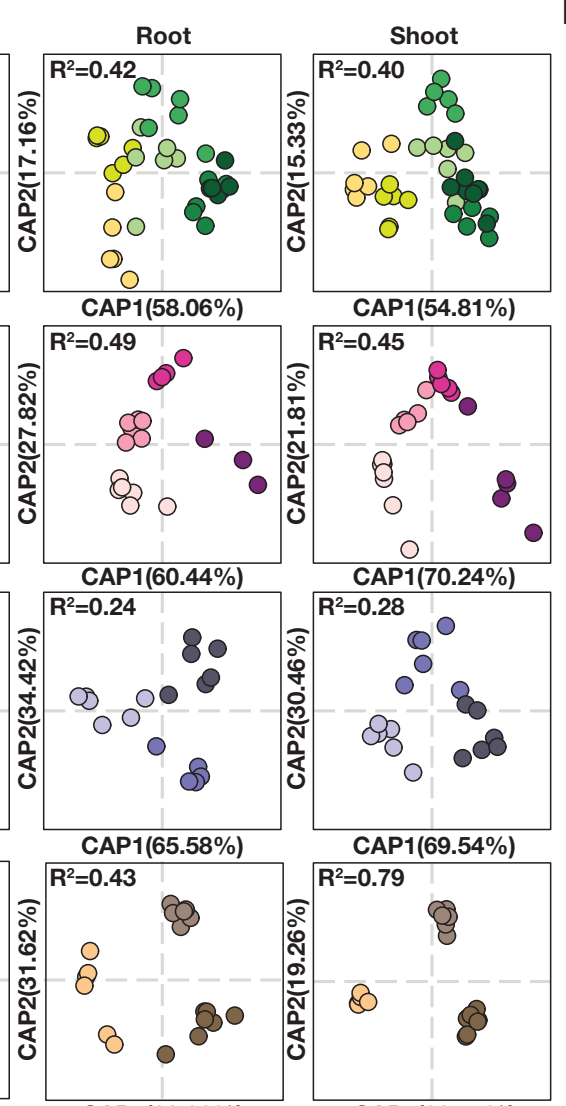

CAP1(68.38\%)
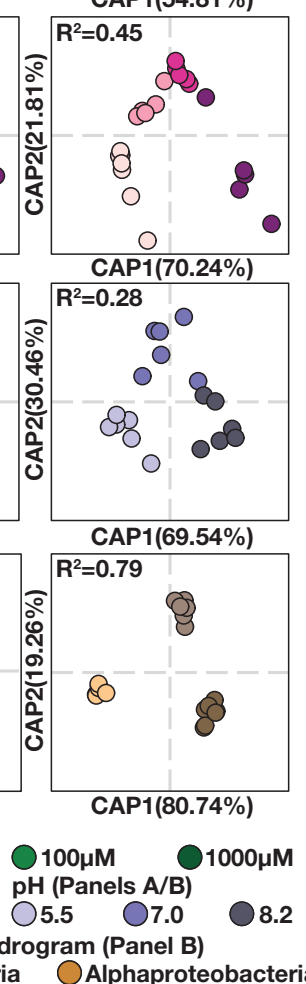

b

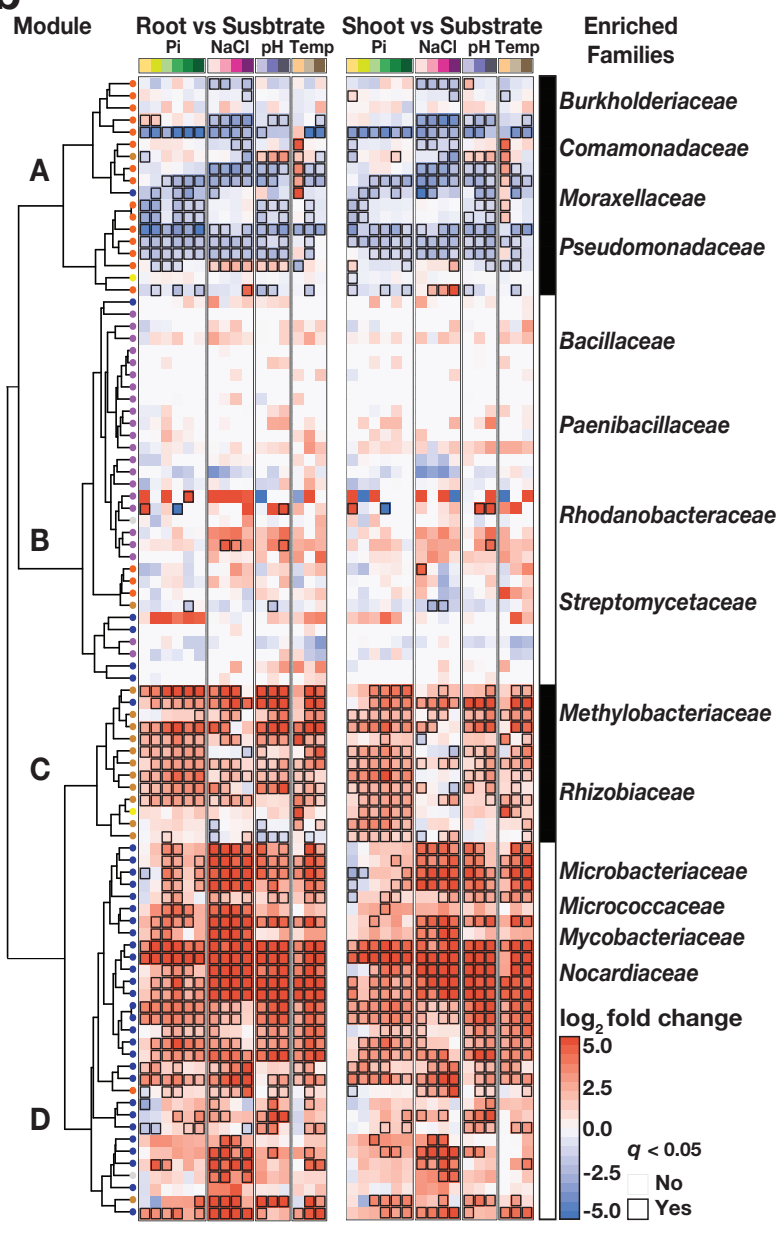

Fig. 1. Reproducible effects of abiotic conditions on the synthetic community assembly.

(a) Canonical analysis of principal coordinates (CAP) scatterplots showing the influence of each of the four abiotic gradients (phosphate, salinity, $\mathrm{pH}$, temperature) within substrate, root and shoot fractions. PERMANOVA R2 values are shown within each plot. (b) Fraction enrichment patterns of the SynCom across abiotic gradients. Each row represents a USeq. Letters on the dendrogram represent the four modules of co-occurring strains (A, B, C, D). Dendrogram tips are colored by taxonomy. The heatmaps are colored by log2 fold changes derived from a fitted GLM. Positive fold changes (red gradient) represent enrichments in plant tissue (root or shoot) compared with substrate, negative fold changes (blue gradient) represent depletion in plant tissue compared with substrate. Comparisons with q-value $<0.05$ are contoured in black. Family bar highlighenriched families within each module. 
bioRxiv preprint doi: https://doi.org/10.1101/645655; this version posted January 16,2020 . The copyright holder for this preprint (which was not certified by peer review) is the author/funder, who has granted bioRxiv a license to display the preprint in perpetuity. It is made available under aCC-BY 4.0 International license.

a

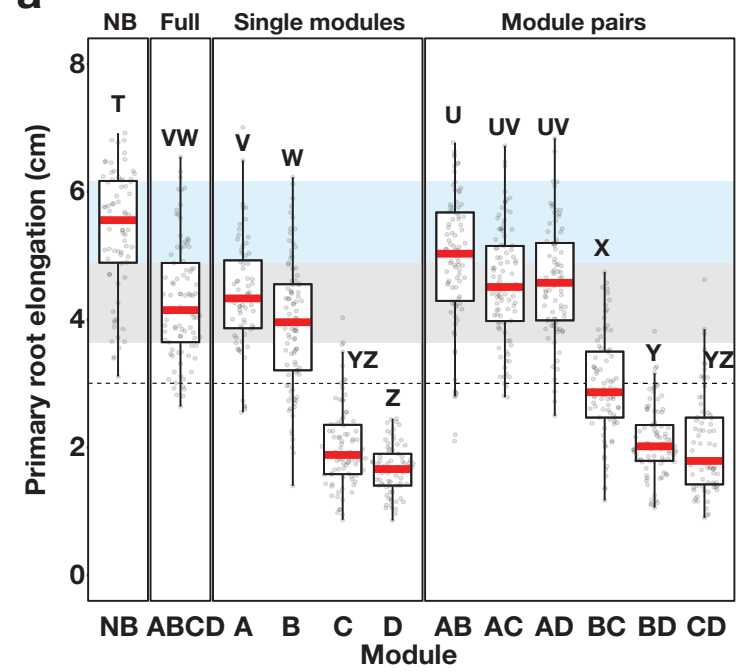

b

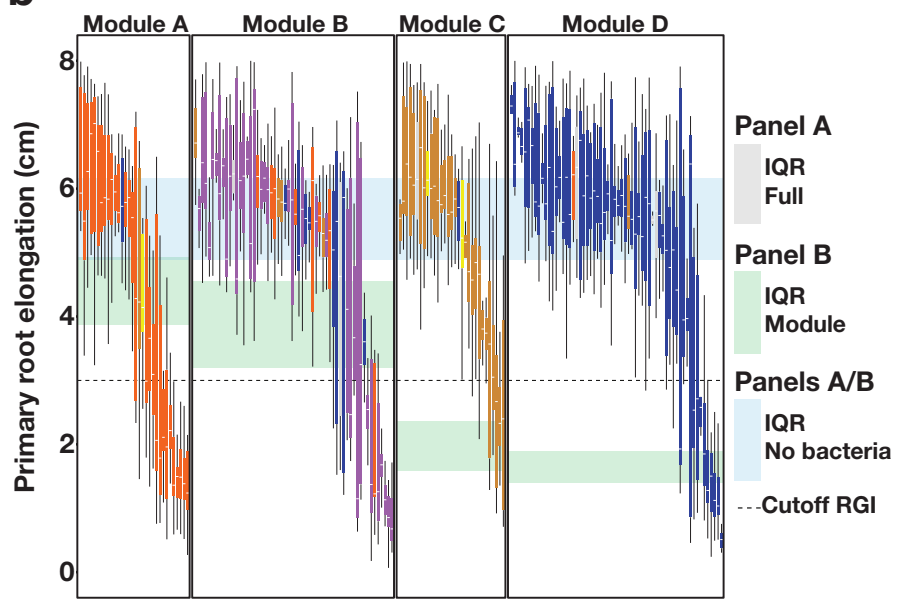

Taxonomy (Panels B/D/E)

Actinobacteria OAlphaproteobacteria OBacteroidetes

OFirmicutes

OGammaproteobacteria

e

Representative RGI reverters

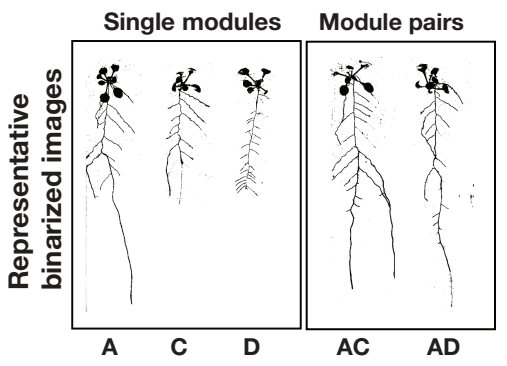

Representative root growth inhibitors

Module A B C D

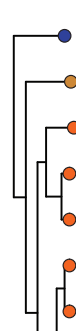

f
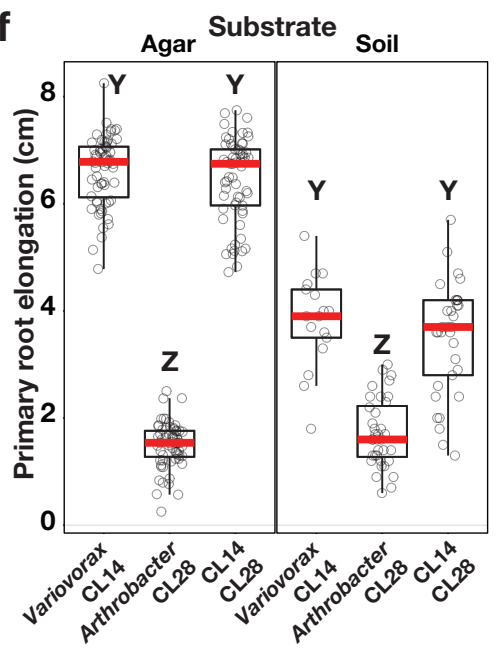

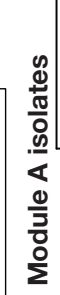

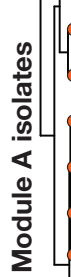

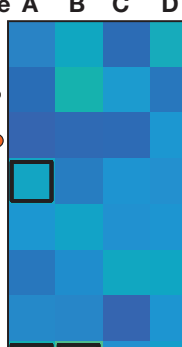

$\square \square$

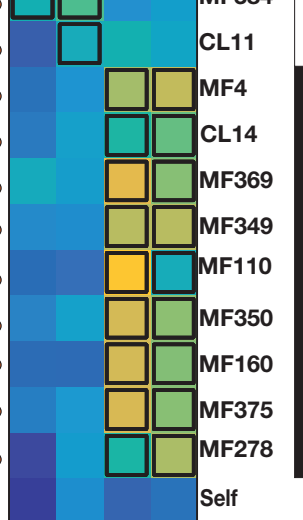

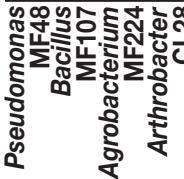

MF455 Mycobacterium

MF220A Sphingomonas

CL71

MF45

MF397

CL144

CL21

MF384

CL11

Burkholderia

.

Panels D/E

Primary root

elongation (cm)

$\begin{array}{llllll} & & & & & \\ 0 & 2 & 4 & 6 & 8\end{array}$

$q<0.05$ \&

estimate $>2$

$\square$ TRUE

Variovorax

范
Module A A A

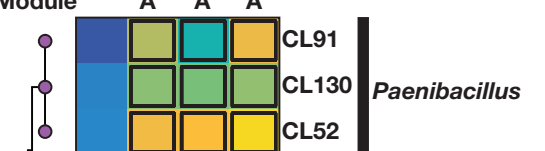

o

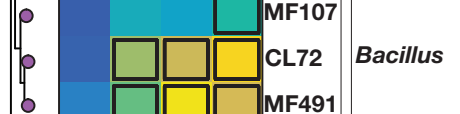

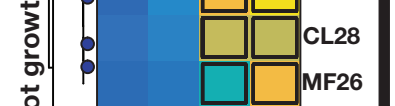

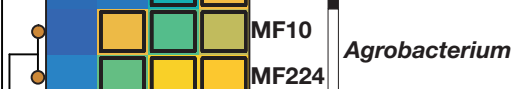

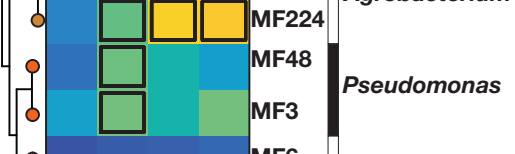

Burkholderia

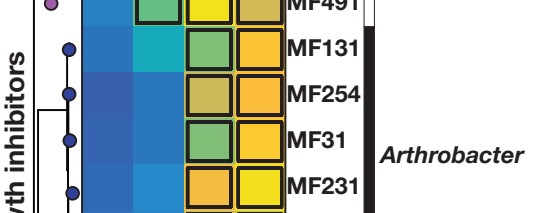


Fig. 2. Arabidopsis root length is governed by multiple bacteria-bacteria interactions within a community.

(a) Primary root elongation of seedlings grown with no bacteria (NB), with the full 185-member SynCom (Full) or with its subsets: Modules A, B, C and D alone (single modules), as well as all six possible pairwise combination of modules (module pairs). Differences between treatments are denoted using the compact letter display. (b) Primary root elongation of seedlings inoculated with single bacterial isolates. Isolates are colored by taxonomy and grouped by module membership. The strips across the panels correspond to the interquartile range (IQR) as noted at far right. The dotted line represents the cutoff used to classify isolates as root growth inhibiting (cutoff RGI). (c) Binarized image of representative seedlings inoculated with modules $A, C$ and $D$, and with module combinations AC and AD. (d, e) Heatmaps colored by average primary root elongation of seedlings inoculated with different pairs of bacterial isolates: (d) with four representative RGl-inducing strains from each module (columns) alone (Self) or in combination with isolates from module A (rows); (e) with eighteen RGl-inducing strains (rows) alone (Self) or in combination with Burkholderia CL11, Variovorax CL14 or Variovorax MF160 (columns). Statistically significant RGI reversions are contoured in black. (f) Primary root elongation of uninoculated seedlings (NB) or seedlings inoculated with Arthrobacter CL28 and Variovorax CL14 isolates individually or jointly. Letters indicate post-hoc significance. 

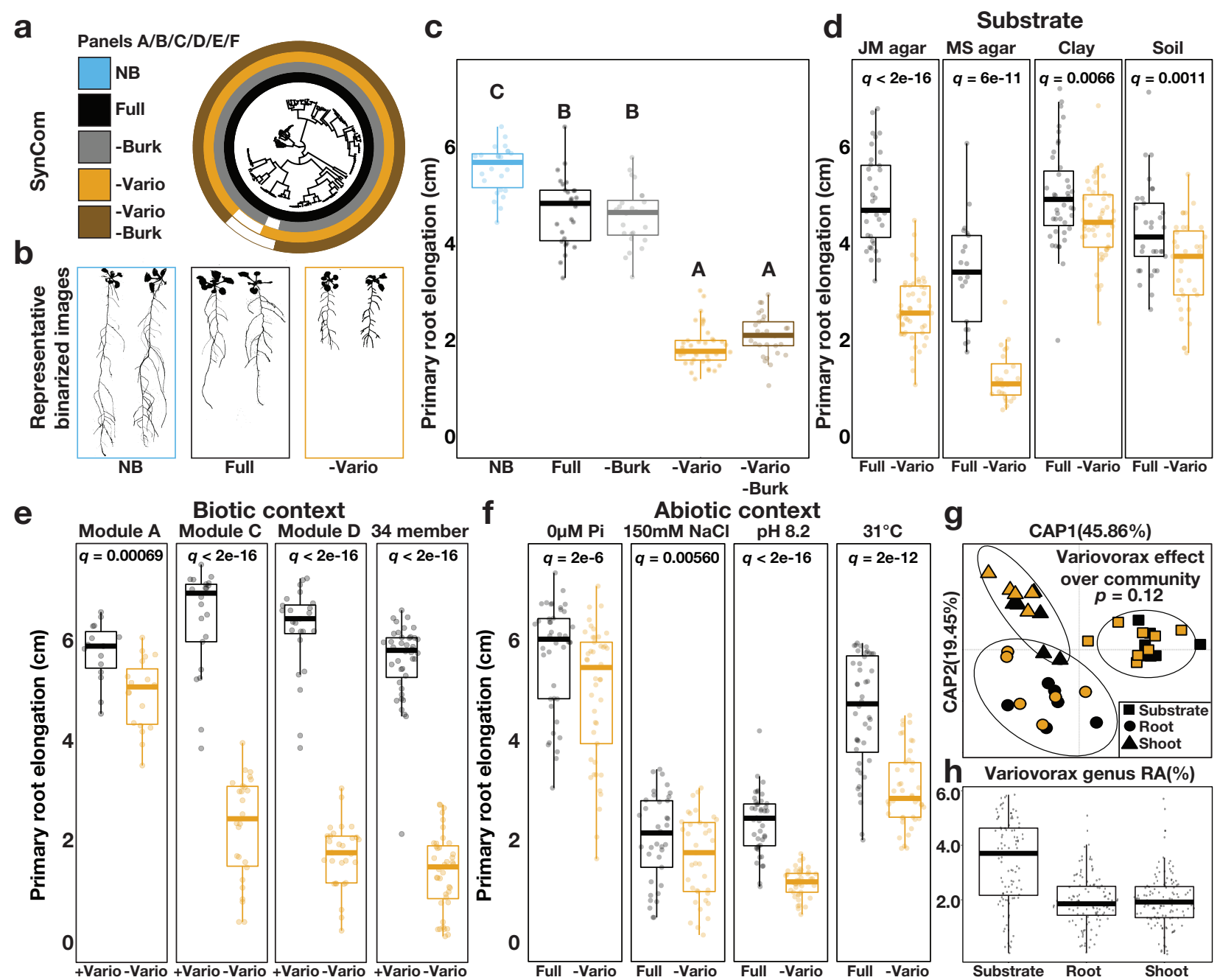

Fig. 3 Variovorax are necessary and sufficient to maintain stereotypic root development.

(a) Phylogenetic tree of 185 bacterial isolates. Concentric rings represent isolate composition of each SynCom treatment (-Burk: Burkholderia drop-out, -Vario: Variovorax drop-out). (b) Binarized image of representative uninoculated seedlings (NB), or seedlings with the full SynCom (Full) or the Variovorax drop-out SynCom (-Vario) treatments. (c) Primary root elongation of uninoculated seedlings (NB) or seedlings with the different SynCom treatments. Letters indicate statistical significance. (d) Primary root elongation of seedlings inoculated with the Full SynCom or with the Variovorax drop-out SynCom (-Vario) across different substrates: Johnson Medium (JM agar), Murashige and Skoog (MS agar), or pots with sterilized clay or potting soil. (e) Primary root elongation of seedlings inoculated independently with four compositionally different SynComs (Module A, C, D and 34-member) with (Full) or without (Vario) 10 Variovorax isolates. (f) Primary root elongation of seedlings inoculated with the Full SynCom or with the Variovorax drop-out SynCom (-Vario) across different abiotic conditions: unamended medium (JM agar control), phosphate starvation (JM agar $0 \mu \mathrm{M} \mathrm{Pi}$ ), salt stress (JM agar $150 \mathrm{mM} \mathrm{NaCl}$ ), high $\mathrm{pH}$ (JM agar $\mathrm{pH}$ 8.2) and high temperature (JM agar $\left.31^{\circ} \mathrm{C}\right)$. FDR-corrected $\mathrm{p}$-values are shown within each plot. (g) Canonical analysis of principal coordinates scatterplots comparing community full vs Variovorax drop-out SynComs across all fractions (agar, root, shoot). PERMANOVA p-value is shown. (h) Relative abundance (RA) of the Variovorax genus within the full SynCom across the agar, root and shoot fractions. 


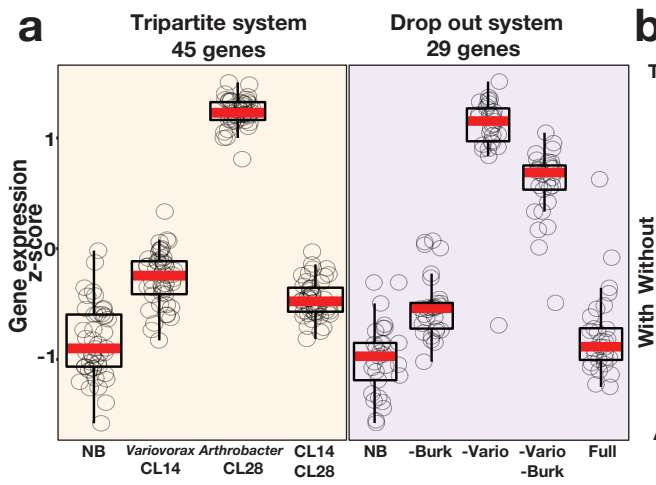

b

c
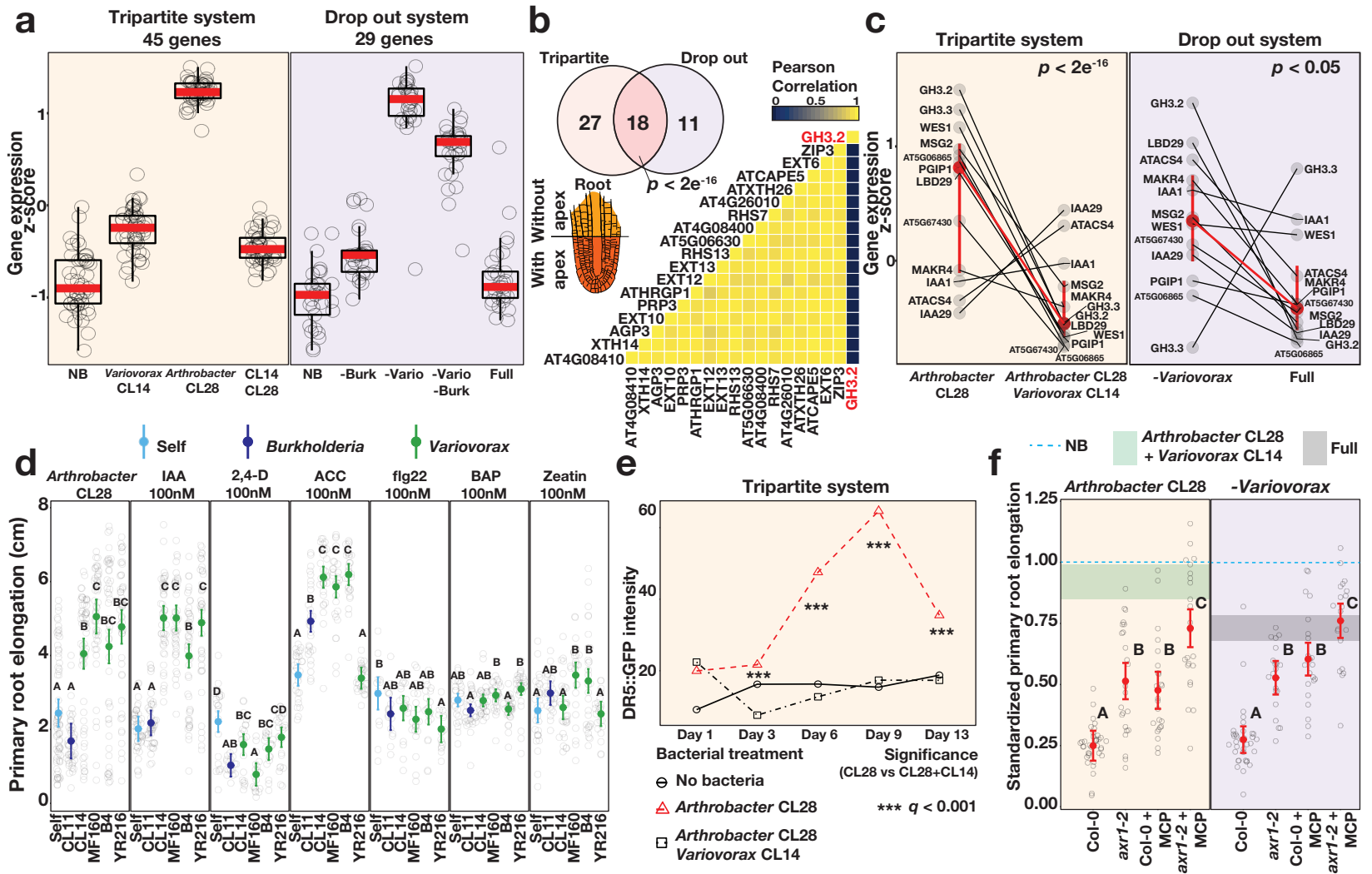

Fig. 4. Variovorax attenuation of root growth inhibition is related to auxin and ethylene signaling.

(a) Boxplots showing the average standardized expression of genes induced in seedlings in response to: Left (Tripartite system) Arthrobacter CL28 compared with uninoculated seedlings (NB) or seedlings inoculated with both Arthrobacter CL28 and Variovorax CL14 (CL14 CL28). Right (Drop-out System) Variovorax drop-out SynCom (-Vario) compared to uninoculated seedlings (NB) and to the full SynCom (Full). (b) Venn diagram showing the overlap of enriched genes between the tripartite and drop-out systems. The heatmap shows the pairwise correlation in expression of these 18 genes across tissues27. (c) Standardized expression of 12 late-responsive auxin genes across the tripartite and drop-out systems. Each dot represents a gene. Identical genes are connected between bacterial treatments with a black line. Mean expression $(95 \% \mathrm{Cl}$ intervals) of the aggregated 12 genes in each treatment is highlighted in red and connected between bacterial treatments with a red line. (d) Primary root elongation of seedlings grown with six hormone or MAMP RGI treatments (panels) individually (Self) or with either Burkholderia CL11 or four Variovorax isolates. Significance between the bacterial treatments is shown using the confidence letter display. (e) GFP intensity of DR5::GFP Arabidopsis seedlings grown with no bacteria, Arthrobacter CL28 and Arthrobacter CL28+Variovorax CL14. Significance within time points is denoted with asterisks. (f) Primary root elongation, standardized to sterile conditions, of wild type (Col-0) auxin unresponsive (axr1-2), ethylene unresponsive (Col-0 + MCP), or auxin/ethylene unresponsive (axr1-2 + MCP) seedlings inoculated with RGI-inducing Arthrobacter CL28 or the Variovorax dropout SynCom (-Variovorax). The blue dotted line marks the relative mean length of uninoculated seedlings. The horizontal shade in each panel corresponds to the interquartile range of seedlings grown with: Arthrobacter CL28+Variovorax CL14), or the full 185-member SynCom including 10 Variovorax isolates (Full SynCom). Differences between treatments are denoted using the compact letter display. 


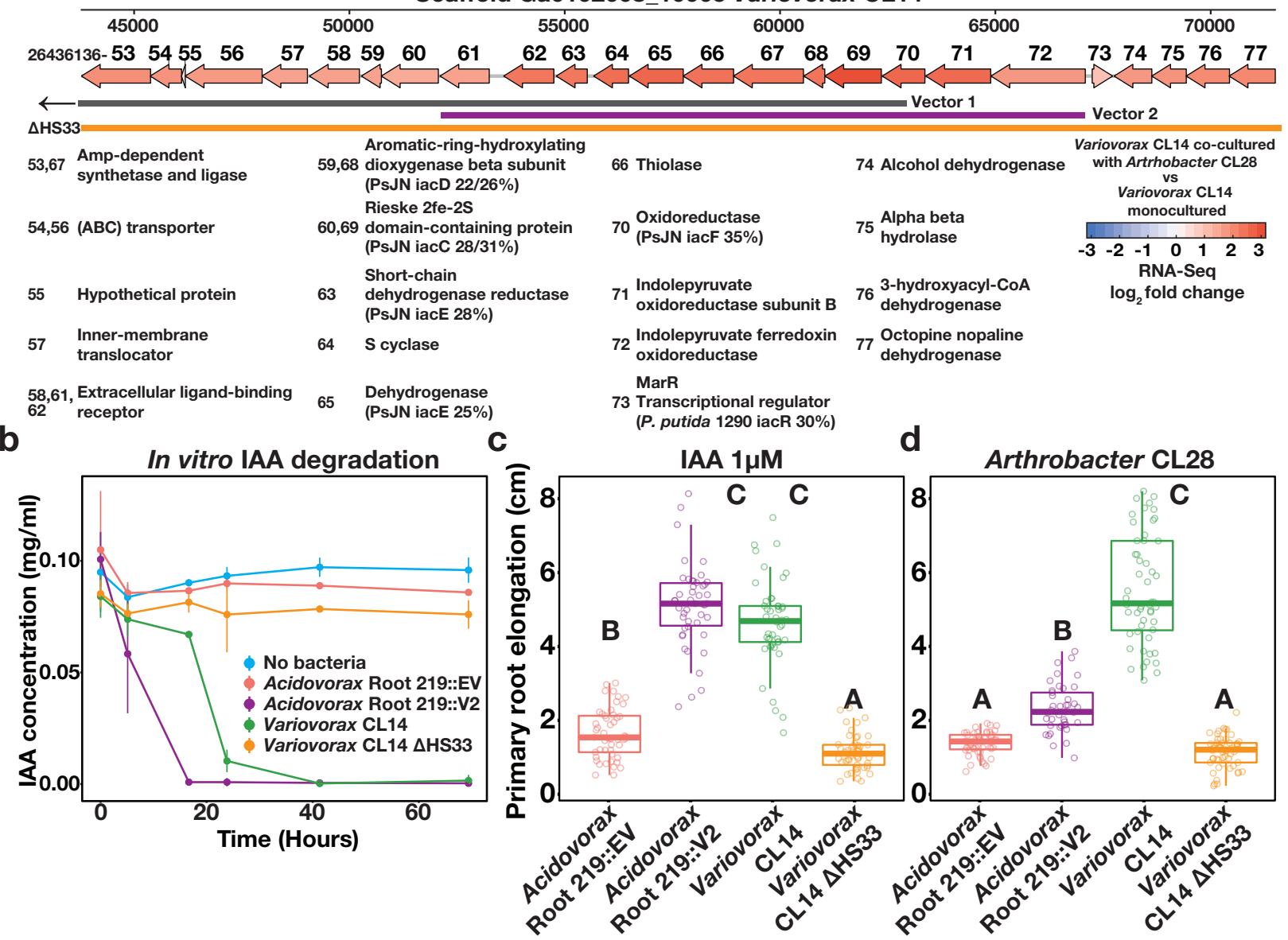

Fig. 5. An auxin-degrading operon in Variovorax is required for maintenance of stereotypical root development

(a) A map of the hotspot 33. Genes are annotated with the last two digits of their IMG gene ID (26436136XX) and their functional assignments are shown below the map, including \% identity of any to genes from a known auxin degradation locus. Gene are colored by the log2 fold change in their transcript abundance in Variovorax CL14 co-cultured with Arthrobacter CL28 vs Variovorax CL14 monoculture. The overlap of this region vectors 1 and 2 and the region knocked out in Variovorax CL14 $\triangle \mathrm{HS} 33$ are shown below the map. Note that vector 1 extends beyond this region. (b) In-vitro degradation of IAA by Acidovorax 219::EV, Acidovorax 219::V2, Variovorax CL14 and Variovorax CL14 $\triangle$ HS33. (c) Primary root elongation of seedlings treated with IAA alone or inoculated with Acidovorax 219::EV, Acidovorax 219::V2, Variovorax CL14 and Variovorax CL14 $\triangle$ HS33. (d) Primary root elongation of seedlings inoculated with Arthrobacter CL28 in alone or together with Acidovorax 219::EV, Acidovorax 219::V2, Variorovax CL14, or Variovorax CL14 $\triangle$ HS33. Letters indicate post-hoc significance. 
bioRxiv preprint doi: https://doi.org/10.1101/645655; this version posted January 16, 2020. The copyright holder for this preprint (which was not certified by peer review) is the author/funder, who has granted bioRxiv a license to display the preprint in perpetuity. It is made available under aCC-BY 4.0 International license.

a

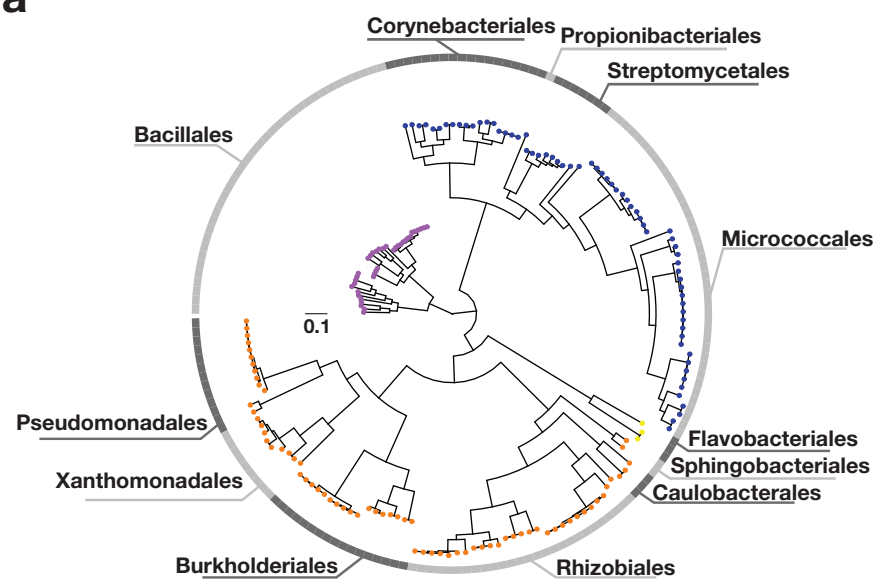

b

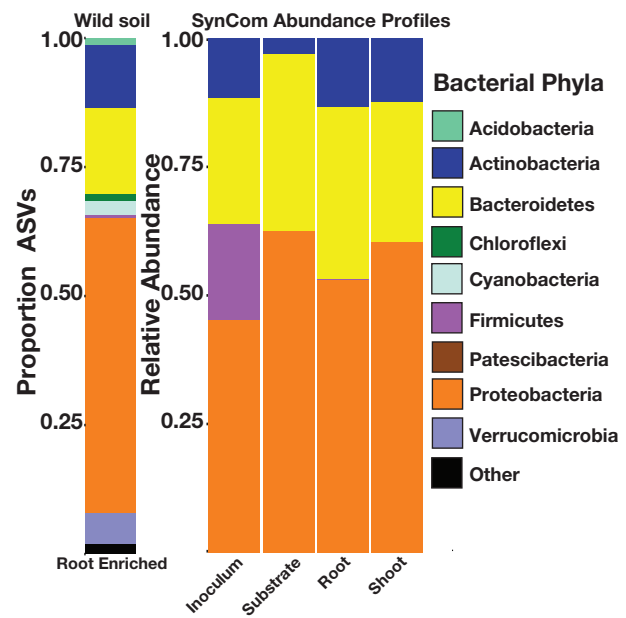

C
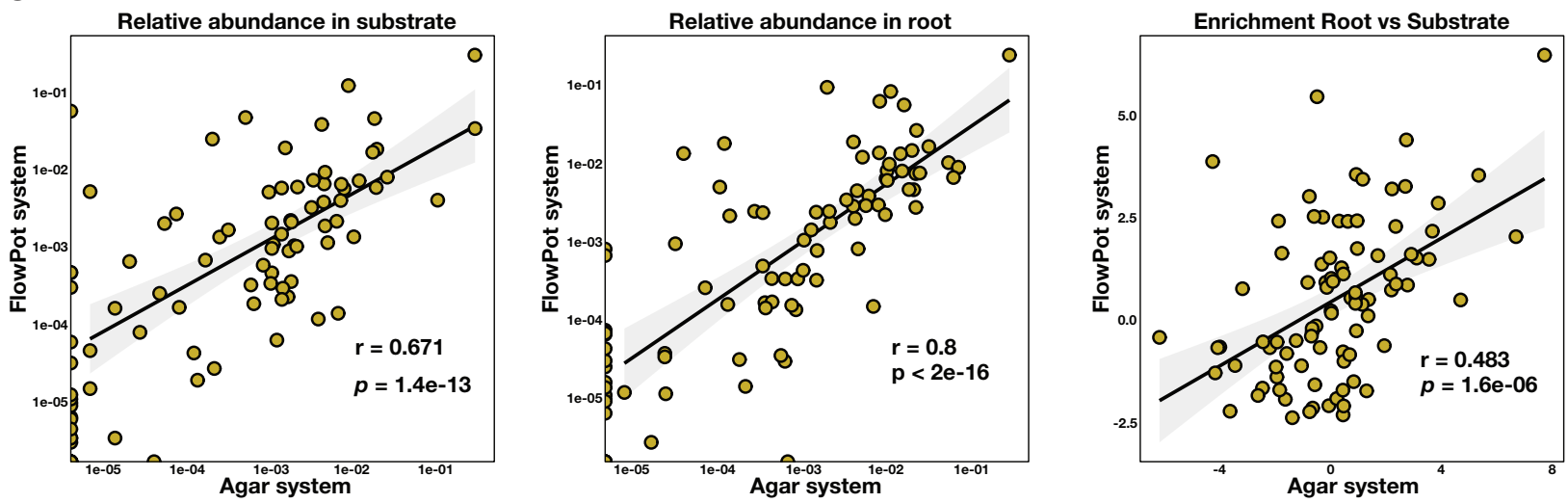

d

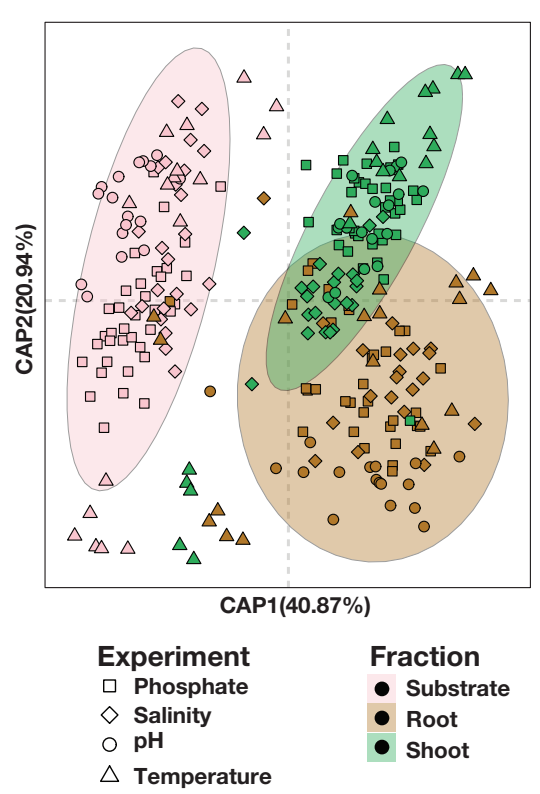

e

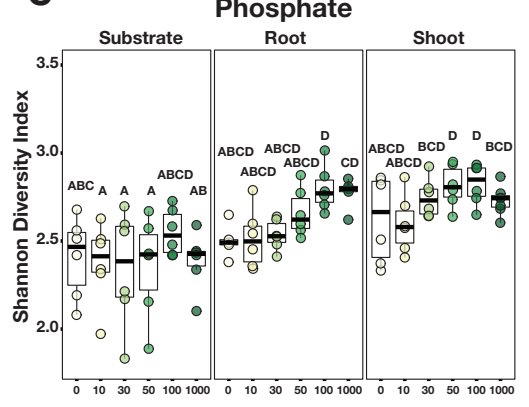

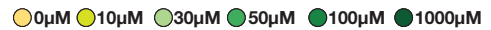

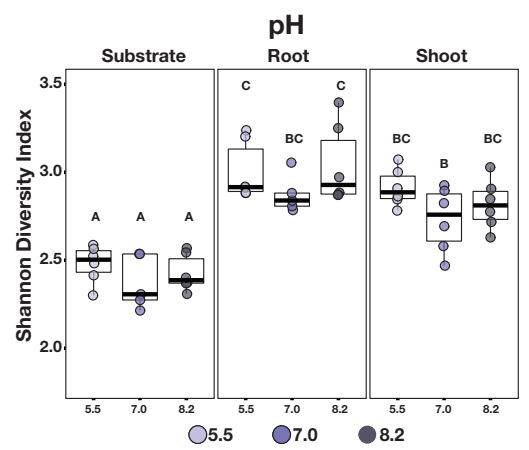

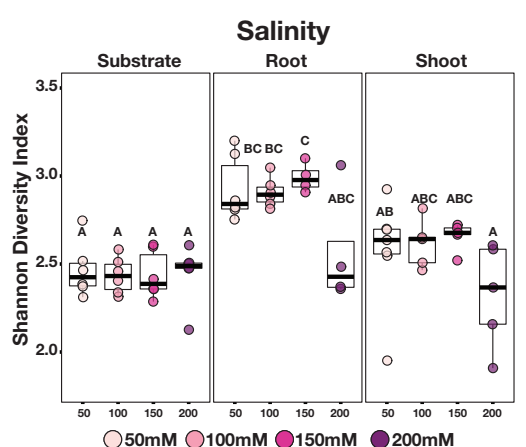

$\bigcirc 50 \mathrm{~mm} \bigcirc 100 \mathrm{~mm} \bigcirc 150 \mathrm{~mm} \bigcirc 200 \mathrm{~mm}$

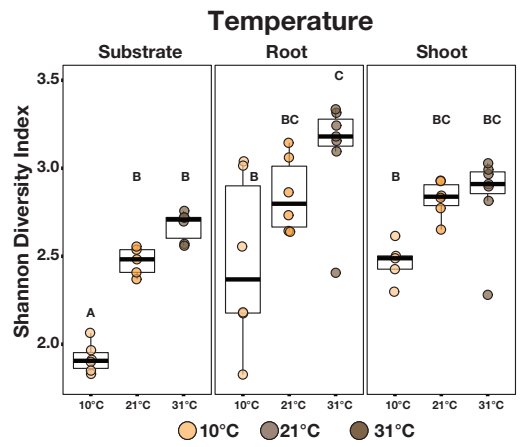




\section{Extended data Fig. 1}

Synthetic community resembles the taxonomic makeup of natural communities.

(a) Phylogenetic tree of 185 bacterial genomes included in the synthetic community (SynCom). The tree tips are colored according to phylum. The outer ring shows the distribution of the 12 distinct bacterial orders present in the SynCom. (b) The panel on the left (wild soil) shows the proportion of amplicon sequence variants (ASVs) enriched (q-value $<0.1)$ in the plant root in comparison to soil in a microbiota profiling study from the same soil that SynCom strains were isolated in Levy et al20. In the panel, ASVs are colored according to phylum and the ASVs belonging to Proteobacteria are colored by class. The panel on the right (SynCom panel) represents the relative abundance profiles of bacterial isolates across the initial inoculum, planted agar, root and shoot in plant exposed to the full SynCom. Bacterial isolates are colored based on their phylum, the Proteobacteria are colored according to the class level classification. (c) Comparison of SynCom community composition in the agar and soil-based microcosms. (Left) Relative abundance in the substrate (FlowPot system) and (middle) root, (right) as well as root vs substrate enrichment levels are shown. Each dot represents a single USeq. Pearson correlation line, 95\% confidence intervals, $r$ value and $p$ value are shown for each comparison. (d) Canonical analysis of principal coordinates showing the influence of the fraction (planted agar, root, shoot) on the assembly of the bacterial SynCom across the four gradients used in this work (phosphate, salinity, $\mathrm{pH}$, temperature). Different colors differentiate between the fractions and different shapes differentiate between experiments. Ellipses denote the $95 \%$ confidence interval of each fraction. (e) Abiotic conditions displayed reproducible effects on alpha-diversity. Each panel represents the bacterial alpha-diversity across the different gradient conditions (phosphate, salinity, $\mathrm{pH}$, temperature) and the fractions (planted agar, root, shoot) used in this work. Bacterial alpha-diversity was estimated using Shannon Diversity. Letters represent the results of the post hoc test of an ANOVA model testing the interaction between fraction and abiotic condition. 
a

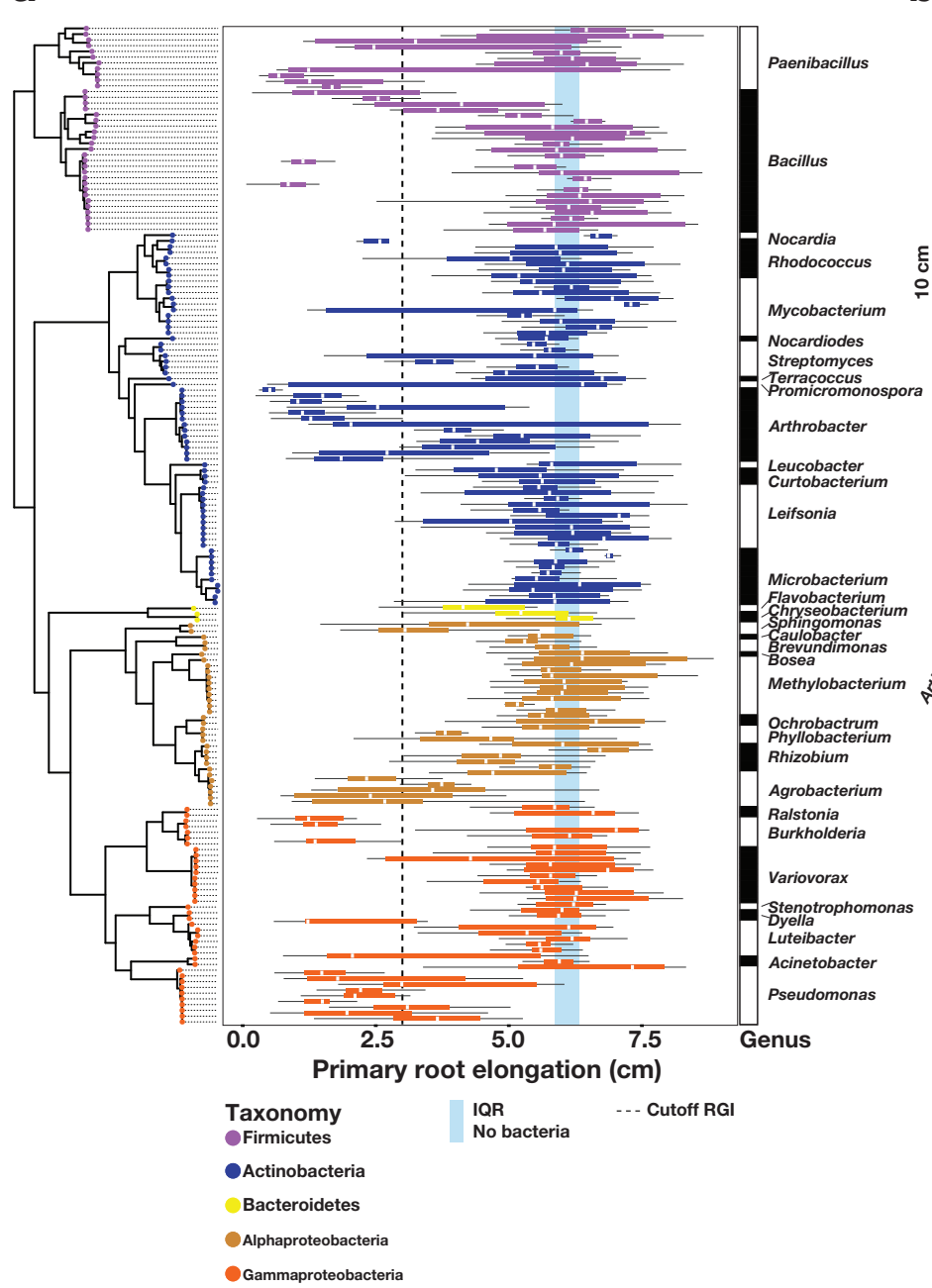

b

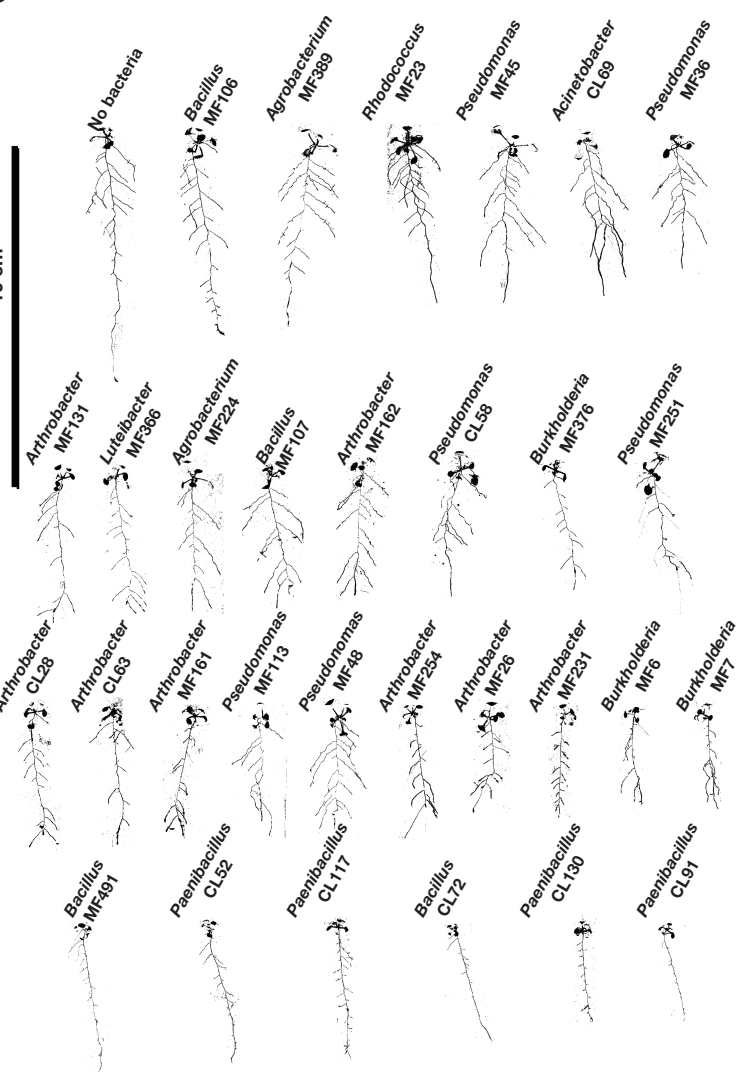

\section{Extended data Fig. 2}

Root growth inhibition trait is distributed across bacterial phylogeny.

(a) Primary root elongation of plants inoculated with single bacterial isolates (one boxplot per isolate). Isolates are ordered according to the phylogenetic tree on the left side of the panel and colored based on their genome-based taxonomy. The vertical blue strips across the panel corresponds to the interquartile range (IRQ) of plants grown in sterile conditions. The vertical dotted line represents the $3 \mathrm{~cm}$ cutoff used to classify strains as root growth inhibiting (RGl) strains. The bar on the right side of the panel denotes the genus classification of each isolate. (b) Binarized image of representative seedlings grown axenically (No bacteria) or with thirty-four RGI strains individually. 

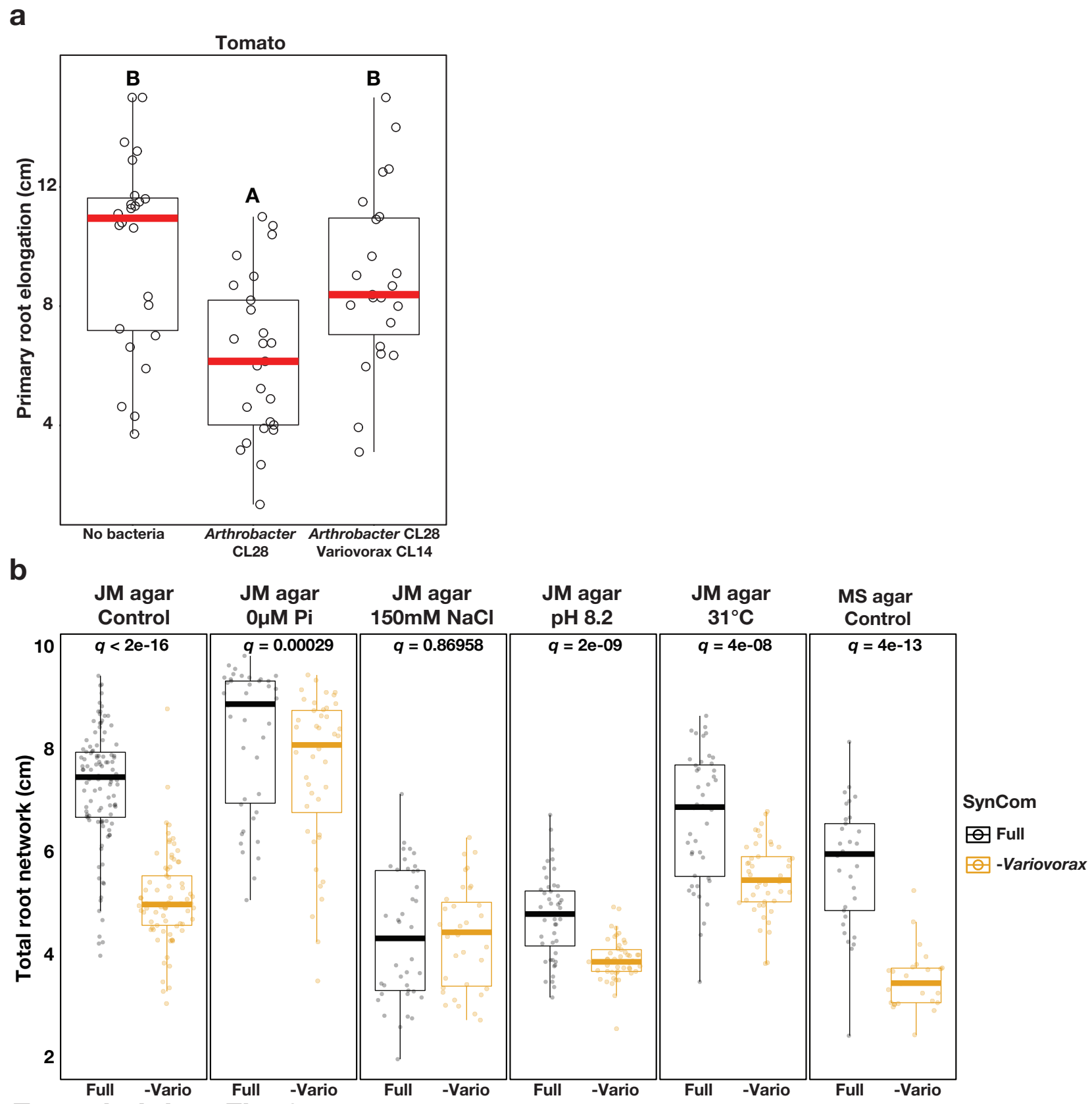

Extended data Fig. 3

\section{Variovorax-mediated reversion of root growth inhibition.}

(a) Variovorax-mediated reversion of root growth inhibition is maintained in a second plant species. Primary root elongation of uninoculated tomato seedlings (No bacteria) or seedlings inoculated with the Arthrobacter CL28 individually or along with Variovorax CL14. Letters indicate post hoc significance. (b) Total root network of Arabidopsis seedlings grown with the full SynCom (Full) or with the full SynCom excluding Variovorax (-Variovorax) across different abiotic conditions: full medium (JM agar control), phosphate starvation (JM agar 0 uM Pi), salt stress (JM agar $150 \mathrm{mM} \mathrm{NaCl}$ ), high $\mathrm{pH}$ (JM agar $\mathrm{pH}$ 8.2) and high temperature (JM agar 31oC) and half Murashige and Skoog medium (MS agar control). Letters indicate statistical significance using ANOVA performed within each experimental condition. 


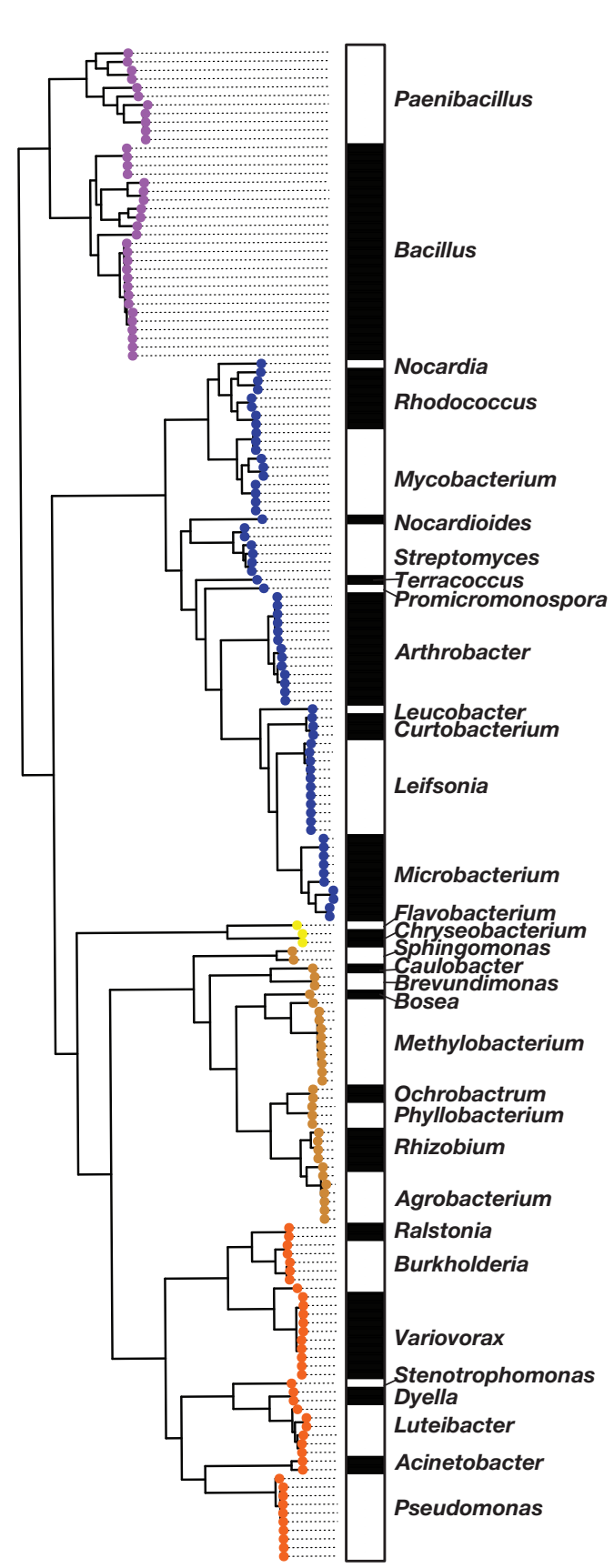

Extended data Fig. 4

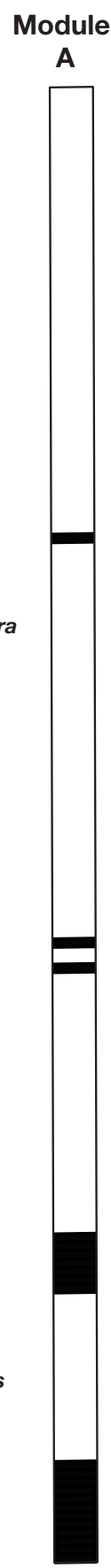

A

Module Module
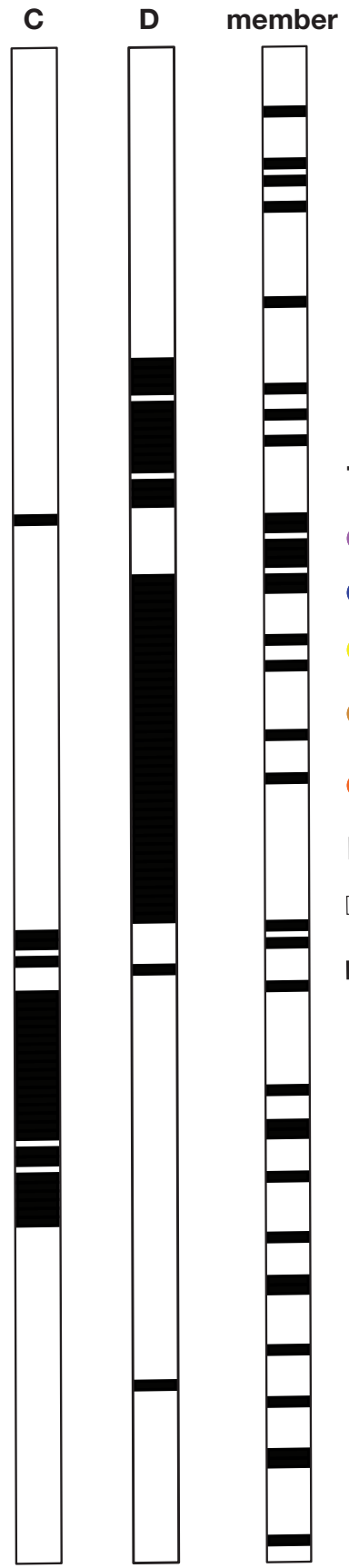

\section{Taxonomy}

Firmicutes

- Actinobacteria

Bacteroidetes

Alphaproteobacteria

Gammaproteobacteria

Inoculation

$\square$ Uninoculated

Inoculated

\section{SynCom}

\section{Taxonomic composition of the SynCom used in Figure 2D.}

Bar graphs showing the isolate composition of SynComs composed by module A (Module A), module C (Module C), module D (Module D) and a 34-member synthetic community (34 member)2. Isolates are ordered according to the phylogenetic tree on the left side of the panel. The tips of the phylogenetic tree are colored based on the genome-based taxonomy of each isolate. Presence of an isolate across the different SynComs is denoted by a black filled rectangle. 
bioRxiv preprint doi: https://doi.org/10.1101/645655; this version posted January 16,2020 . The copyright holder for this preprint (which was not certified by peer review) is the author/funder, who has granted bioRxiv a license to display the preprint in perpetuity. It is made available under aCC-BY 4.0 International license.

a

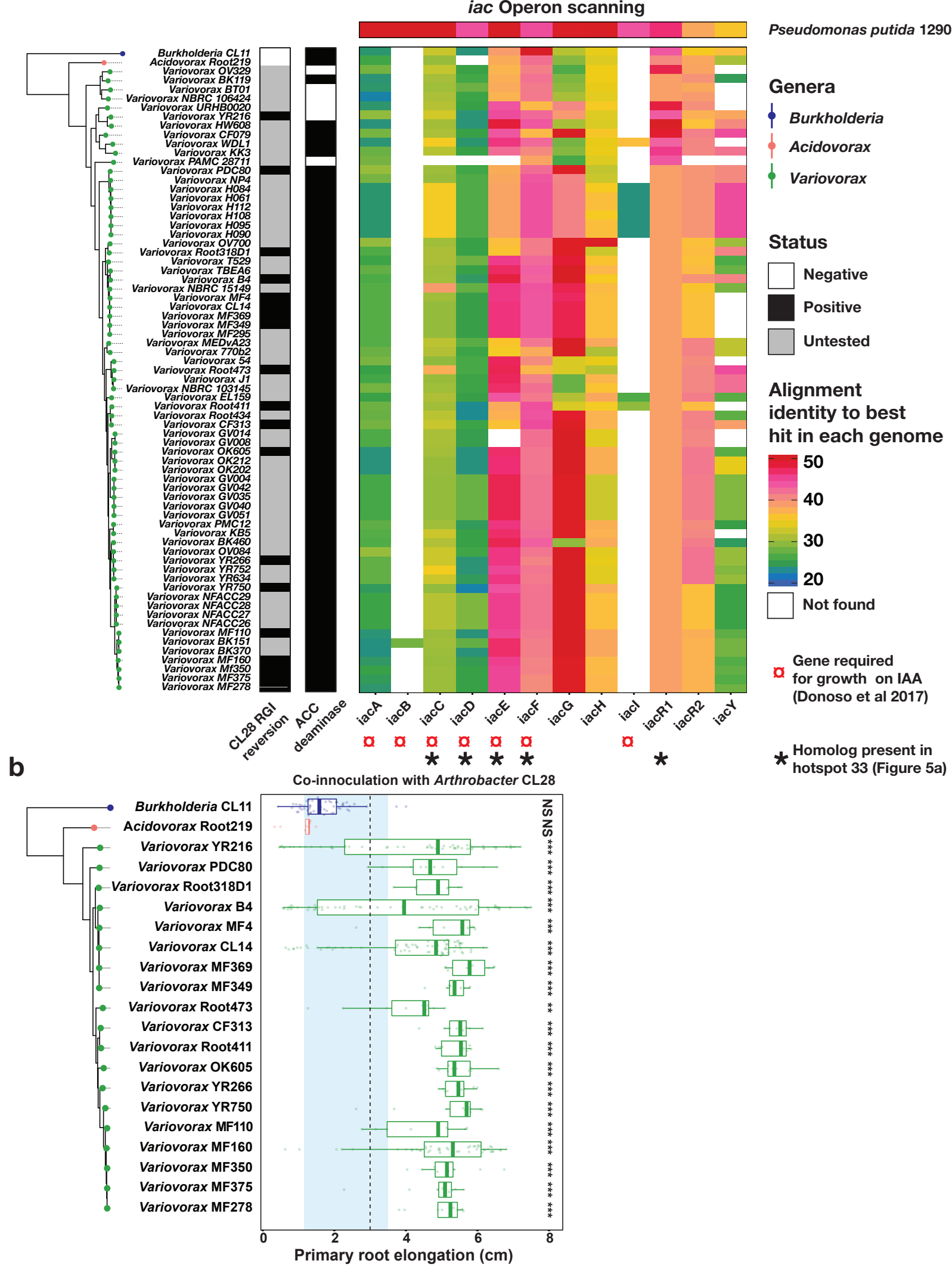




\section{Extended data Fig. 5}

Reversion of root growth inhibition is prevalent across the Variovorax phylogeny. (a) Phylogenetic tree of 54 publically available Variovorax genomes and two outgroup isolates, Acidovorax Root219 and Burkholderia CL11. The CL28 RGI reversion bar binarizes (positive, negative, untested) the ability of each isolate in the phylogeny to revert the root growth inhibition caused by Arthrobacter CL28. The ACC deaminase bar denotes the presence of the KEGG orthology term KO1505 (1-aminocyclopropane-1-carboxylate deaminase) in each of the genomes. The heatmap denotes the percent identity of BLASTp hits in the genomes to the genes from the auxin degrading iac operon in Paraburkholderia phytophirmans described by Donoso, et al.33. Note that synteny is not conserved and these BLAST hits are spread throughout the genomes. (b) Phylogenetic tree of 19 Variovorax genomes along two outgroup isolates, Acidovorax Root219 and Burkholderia CL11 that were tested for their ability to revert the root growth inhibition (RGI) imposed by Arthrobacter CL28. The blue vertical strip across the panel denotes the interquartile range of plants treated solely with Arthrobacter CL28. The dotted vertical line across the panel denotes the $3 \mathrm{~cm}$ cutoff used to classify a treatment as a root growth inhibitor (RGI). Each boxplot is colored according to the genus classification of each isolate. Statistical significance is denoted on the top of each boxplot. 


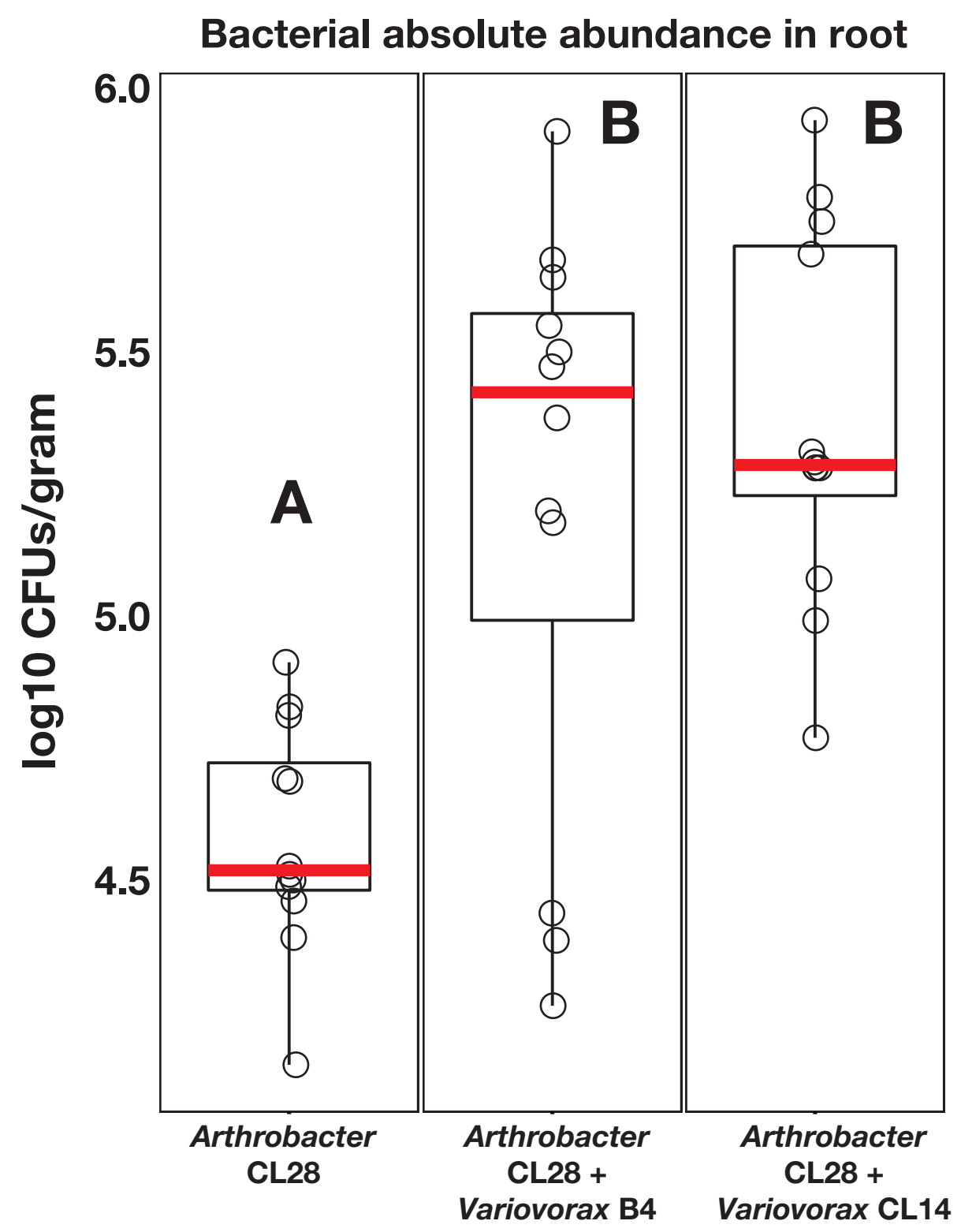

Extended data Fig. 6

Variovorax does not inhibit the growth of an RGI strain. In planta absolute abundance of Arthrobacter CL28 when inoculated alone or with two Variovorax representatives: Variovorax B4 and Variovorax CL14. Log-transformed-Colony forming Units (CFU) of Arthrobacter CL28 normalized to root weight are shown. To selectively grow Arthrobacter CL28, CFUs were counted on Luria Bertani (LB) agar plates containing $50 \mu \mathrm{g} / \mathrm{ml}$ of Apramycin, on which both Variovorax B4 and Variovorax CL14 do not grow. 


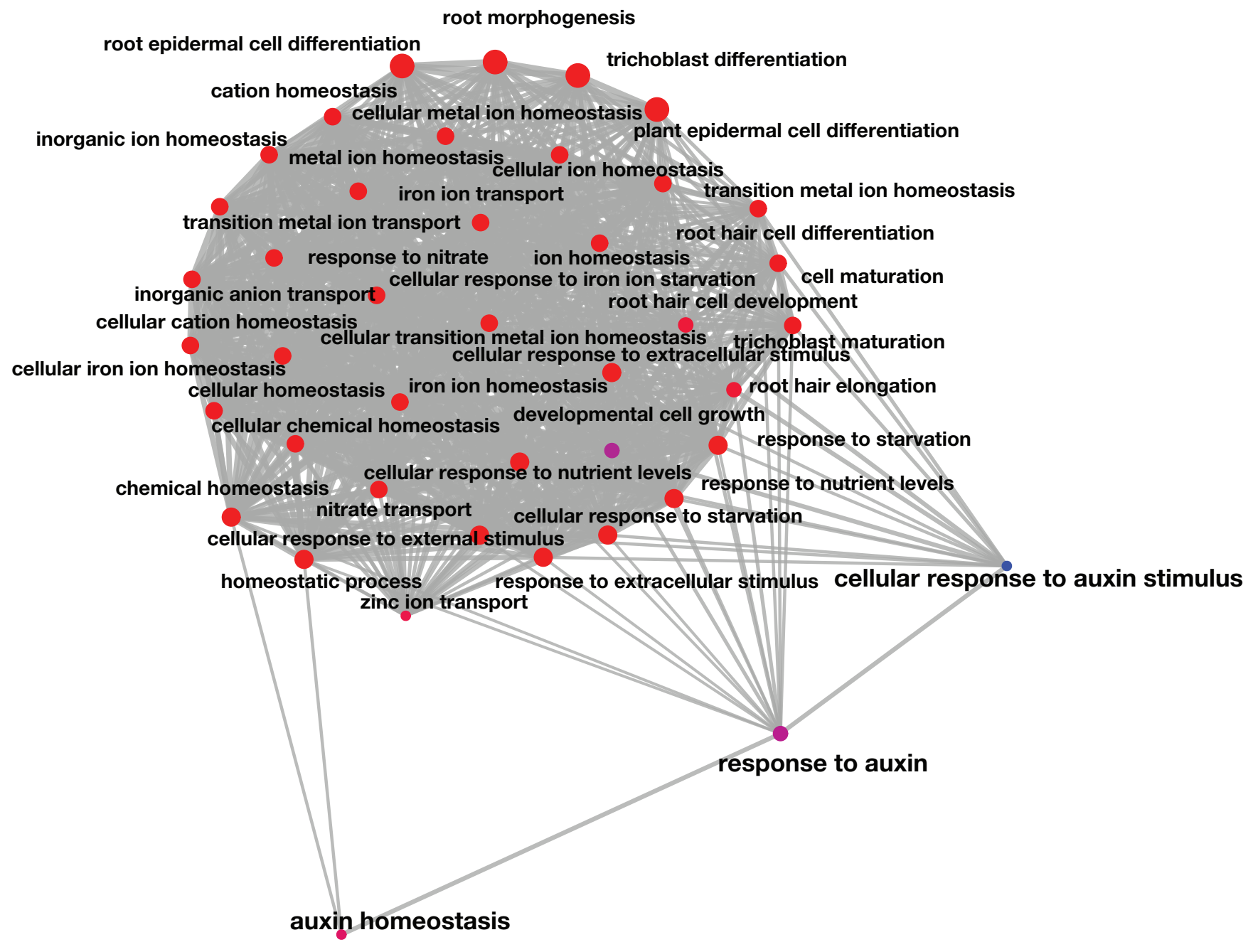

\section{Extended data Fig. 7}

Root growth inhibition-related genes share gene ontologies.

Network of statistically significant gene ontology terms contained in the 18 genes upregulated in Variovorax CL14/Arthrobacter CL28 co-inoculation vs Arthrobacter CL28 alone AND in the full SynCom vs the Variovorax drop-out SynCom (See Figure 4a and 4b). The network was computed using the emapplot function from the package clusterProfiler in R. A p-value for terms across the gene ontology was computed using a hypergeometric test, additionally the size of each point (Gene ontology term) denoted the number of genes mapped in that particular term. 
a

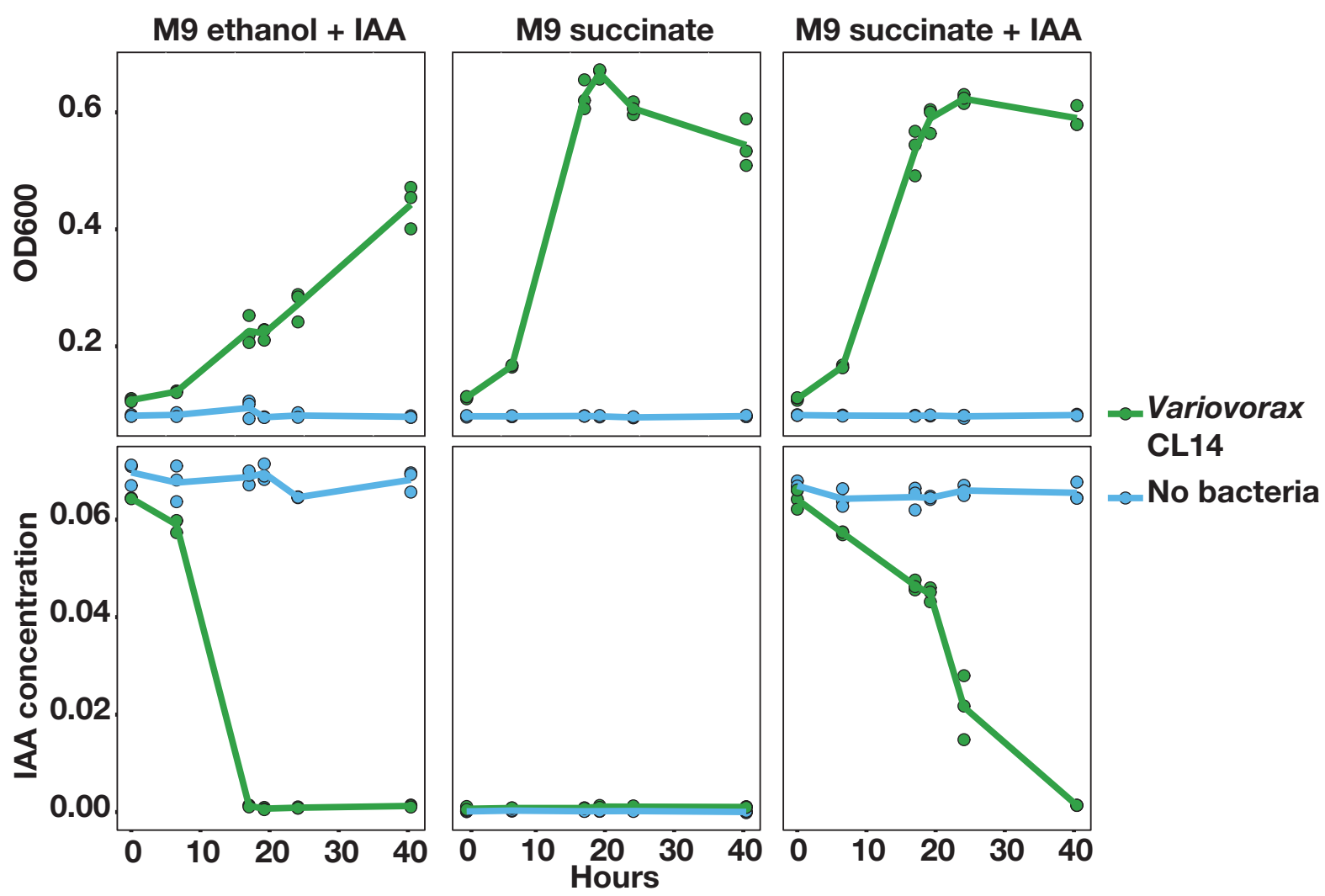

b

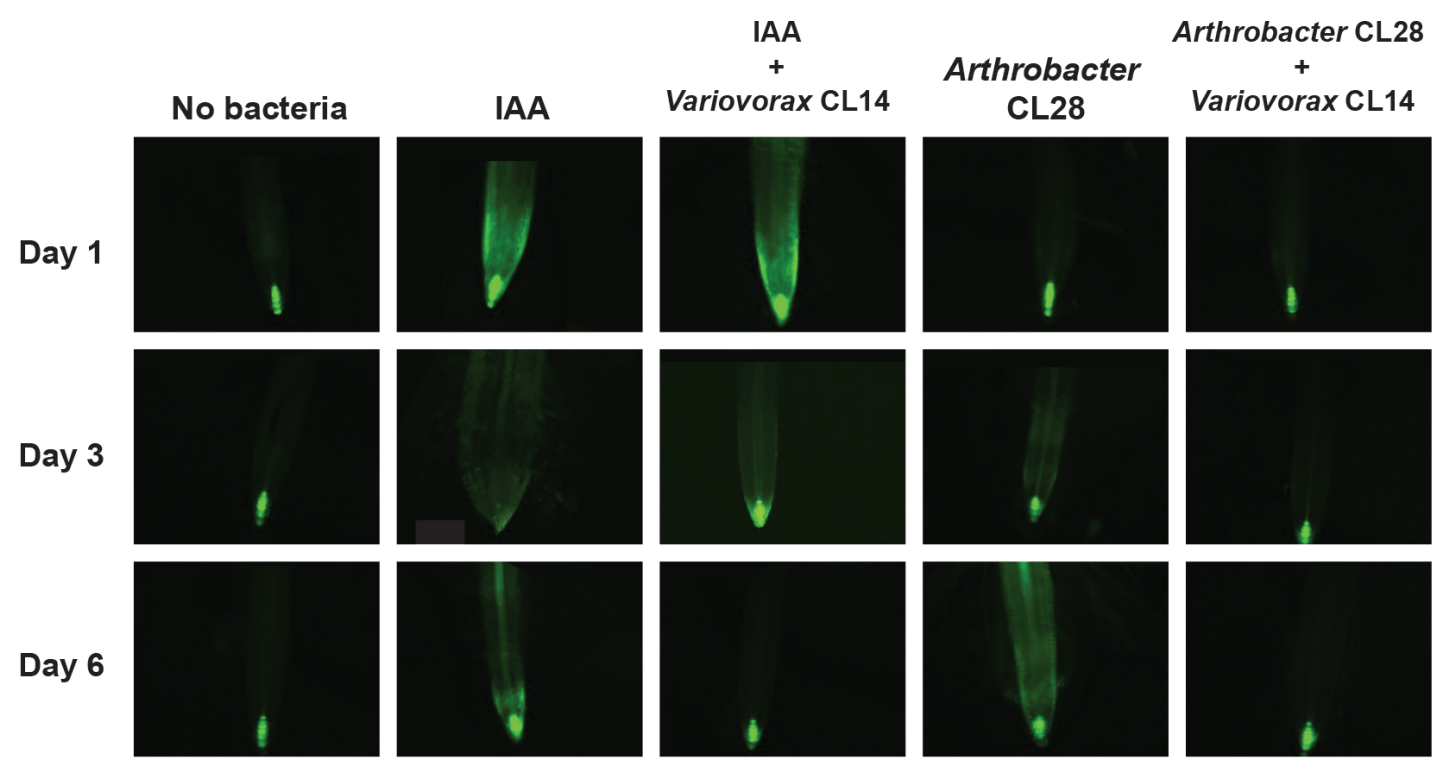




\section{Extended data Fig. 8}

Variovorax degrades auxin and quenches auxin perception by the plant.

(a) Variovorax utilizes auxin as a carbon source. Growth curves showing Optical density at OD600 (top) and Indole-3-acetic acid (IAA) concentrations (mg/mL bottom) in Variovorax CL14 cultures grown in M9 media with carbon sources: IAA and ethanol (from IAA solubilization) (left), succinate (center), and succinate, IAA, and ethanol (right). (b) Variovorax quenches auxin bioreporter DR5::GFP induction. Main root tips of DR5::GFP plants grown with different Indole-3-acetic acid (IAA) and bacterial treatments. DR5::GFP plants were treated with the tripartite system (Arthrobacter CL28, Variovorax CL14, CL28+CL14), with the drop-out System (full Syncom, Variovorax drop-out Syncom [-Variovorax]), IAA and IAA+Variovorax CL14. GFP fluorescence was imaged 1, 3 and 6 days post inoculation. Fluorescence was quantified in the root elongation zone. 
a

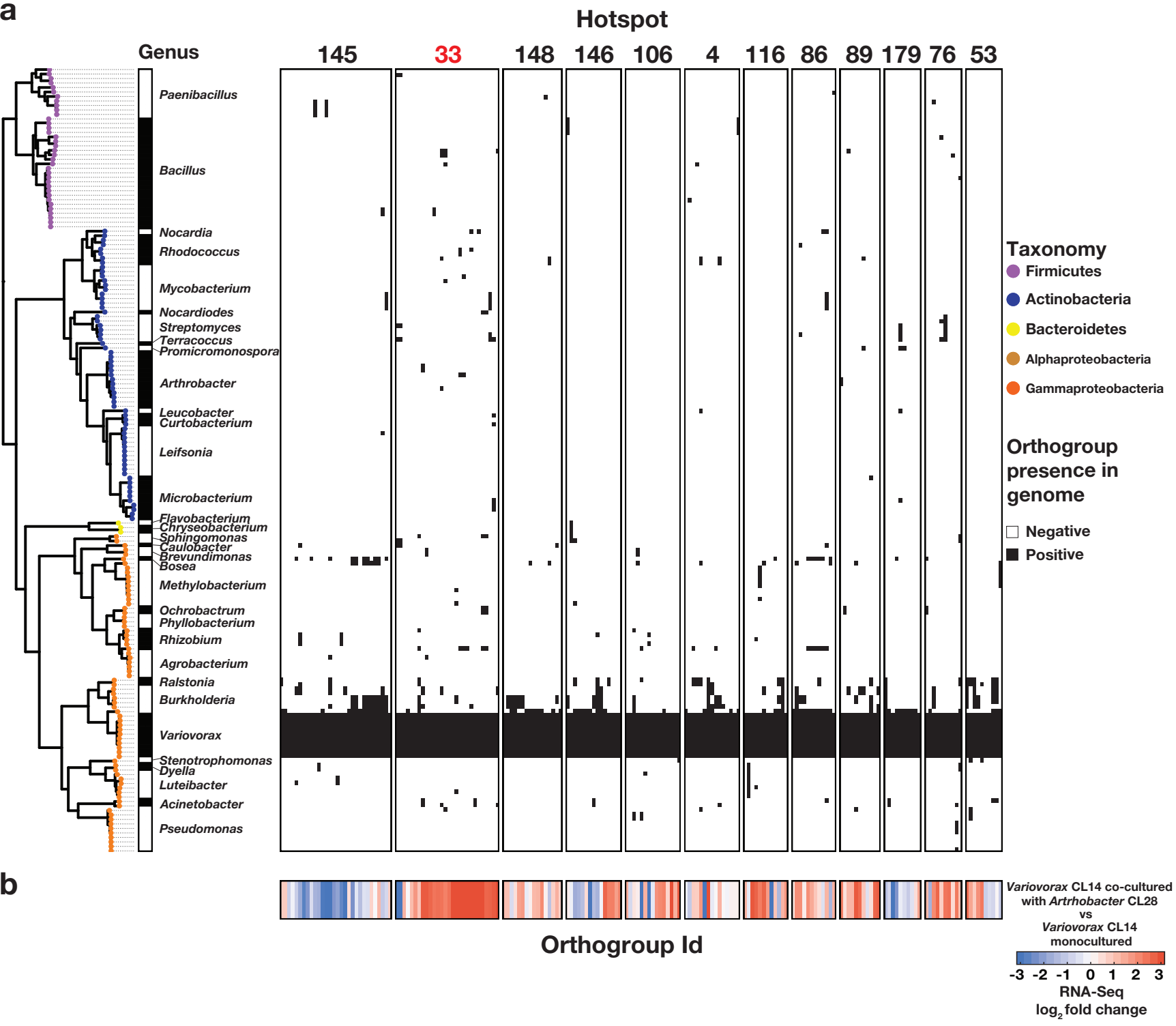

b

Extended Data Fig. 9.

Detection of CL28-responsive Variovorax-unique operons.

(a) Presence/absence matrix denoting the distribution of 12 Variovorax-unique hotspots containing at least 10 genes across the 185 members of the SynCom. Hotspots are defined using the Variovorax CL14 genome as a reference. Phylogeny of the 185 SynCom members is shown to the left of the matrix. We determined the presence of an orthogroup based on a hidden Markov model profile scanning of each orthogroup across the 185 genomes in the SynCom. (b) Results of bacterial RNA-Seq. Log2(fold change) of each gene shown in the matrix in Variovorax CL14 is co-cultured with Arthrobacter CL28 versus Variovorax CL14 monoculture. Note uniform up-regulation of genes in cluster 33. 


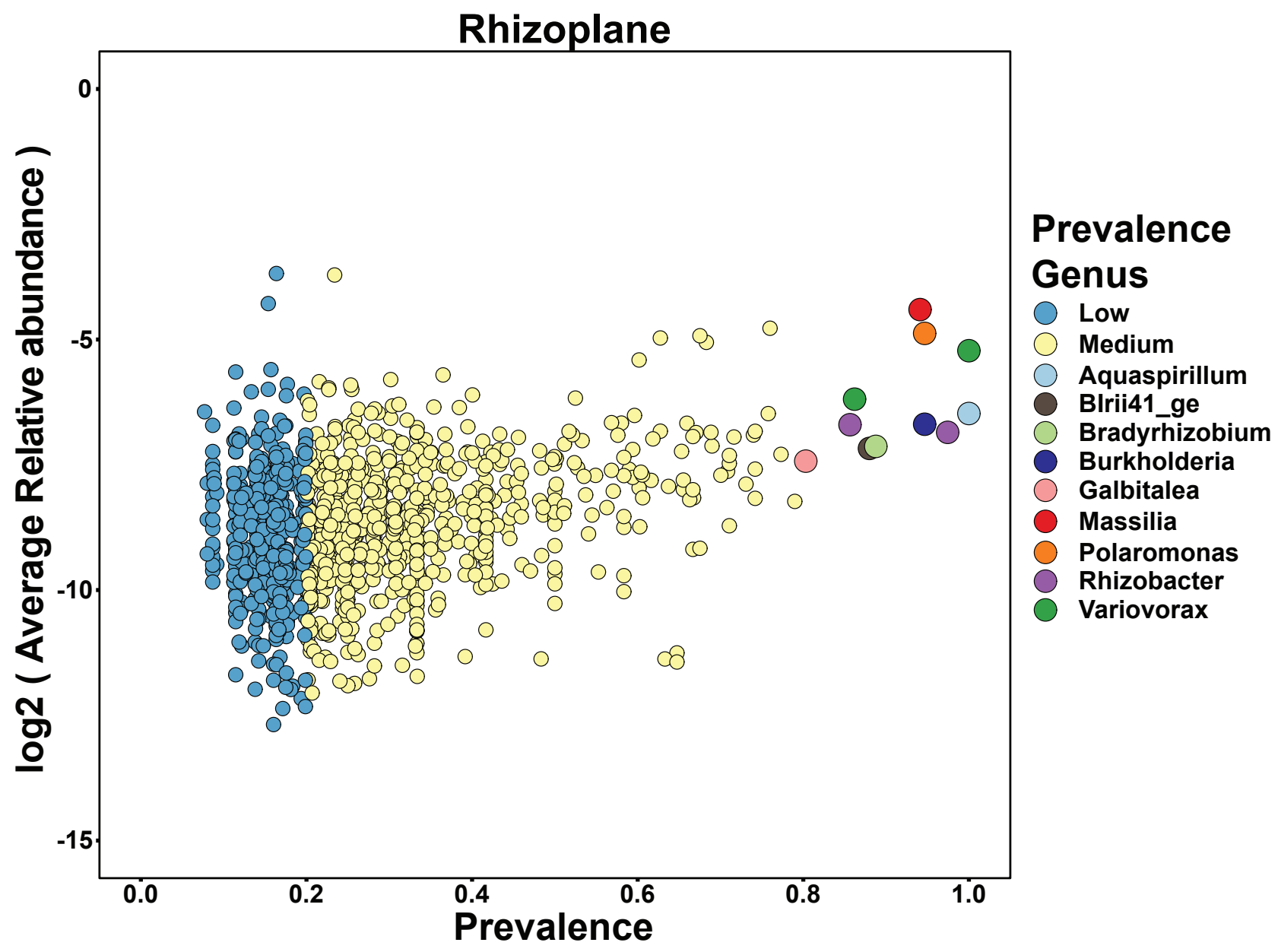

\section{Extended data Fig. 10}

Variovorax are highly prevalent across naturally occurring Arabidopsis microbiomes.

Correlation plot of data reanalyzed from Thiegart et al38 comparing bacterial amplicon sequence variants (ASV) prevalence to log transformed relative abundance in Arabidopsis thaliana rhizosphere samples taken across 3 years in 17 sites in Europe. 


\section{Methods}

295 1. Arabidopsis with bacterial SynCom microcosm across four stress gradients (Fig. 1,

296 fig. S2-S3, data S2)

297 a. Bacterial culture and plant-inoculation

298 The 185-member bacterial synthetic community (SynCom) used here contains genome299 sequenced isolates obtained from surface sterilized Brassicaceae roots, nearly all Arabidopsis thaliana, planted in two North Carolina, US, soils. A detailed description of 301 this collection and isolation procedures can be found $\mathrm{in}^{24}$. One week prior to each

302 experiment, bacteria were inoculated from glycerol stocks into $600 \mu \mathrm{L} \mathrm{KB}$ medium in a 30396 deep well plate. Bacterial cultures were grown at $28^{\circ} \mathrm{C}$, shaking at $250 \mathrm{rpm}$. After five 304 days of growth, cultures were inoculated into fresh media and returned to the incubator 305 for an additional 48 hours, resulting in two copies of each culture, 7 days old and 48 306 hours old. We adopted this procedure to account for variable growth rates of different 307 SynCom members and to ensure that non-stationary cells from each strain were 308 included in the inoculum. After growth, 48-hour and 7-day plates were combined and 309 optical density of cultures was measured at $600 \mathrm{~nm}\left(\mathrm{OD}_{600}\right)$ using an Infinite M200 Pro 310 plate reader (TECAN). All cultures were then pooled while normalizing the volume of each 311 culture to $\mathrm{OD}_{600}=1$. The mixed culture was washed twice with $10 \mathrm{mM} \mathrm{MgCl} 2$ to remove 312 spent media and cell debris and vortexed vigorously with sterile glass beads to break up 313 aggregates. $\mathrm{OD}_{600}$ of the mixed, washed culture was then measured and normalized to $314 \mathrm{OD}_{600}=0.2$. The SynCom inoculum $(100 \mu \mathrm{L})$ was spread on $12 \times 12 \mathrm{~cm}$ vertical square 315 agar plates with amended Johnson medium $(\mathrm{JM})^{2}$ without sucrose prior to transferring seedlings. 
b. In vitro plant growth conditions

All seeds were surface-sterilized with $70 \%$ bleach, $0.2 \%$ Tween-20 for $8 \mathrm{~min}$, and rinsed

surface. Seeds were stratified at $4{ }^{\circ} \mathrm{C}$ in the dark for two days. Plants were germinated

Then, 10 plants were transferred to each of the SynCom-inoculated agar plates. The composition of $\mathrm{JM}$ in the agar plates was amended to produce environmental variation.

We added to the previously reported phosphate concentration gradient $(0,10,30,50$,

was tested separately, in two independent replicas. Each condition included three

placed in randomized order in growth chambers and grown under a 16-h dark/8-h light regime at $21{ }^{\circ} \mathrm{C}$ day/ $18{ }^{\circ} \mathrm{C}$ night for 12 days. Upon harvest, DNA was extracted from roots, shoots and agar.

c. DNA extraction

335 Roots, shoots and agar were harvested separately, pooling 6-8 plants for each sample.

336 Roots and shoots were placed in $2 \mathrm{~mL}$ Eppendorf tubes with three sterile glass beads.

337 These samples were washed three times with sterile distilled water to remove agar 338 particles and weakly associated microbes. Tubes containing the samples were stored at $339-80{ }^{\circ} \mathrm{C}$ until processing. Root and shoot samples were lyophilized for 48 hours using a 
340 Labconco freeze dry system and pulverized using a tissue homogenizer (MPBio). Agar

341 from each plate was collected in $30 \mathrm{~mL}$ syringes with a square of sterilized Miracloth

342 (Millipore) at the bottom and kept at $-20^{\circ} \mathrm{C}$ for one week. Syringes were then thawed at

343 room temperature and samples were squeezed gently through the Miracloth into $50 \mathrm{~mL}$

344 falcon tubes. Samples were centrifuged at max speed for $20 \mathrm{~min}$ and most of the

345 supernatant was discarded. The remaining 1-2 $\mathrm{mL}$ of supernatant, containing the pellet,

346 was transferred into clean $1.5 \mathrm{~mL}$ Eppendorf tubes. Samples were centrifuged again,

347 supernatant was removed, and pellets were stored at $-80{ }^{\circ} \mathrm{C}$ until DNA extraction. DNA

348 extractions were carried out on ground root and shoot tissue and agar pellets using 96-

349 well-format MoBio PowerSoil Kit (MOBIO Laboratories; Qiagen) following the

350 manufacturer's instruction. Sample position in the DNA extraction plates was

351 randomized, and this randomized distribution was maintained throughout library

352 preparation and sequencing.

355 We amplified the V3-V4 regions of the bacterial 16S rRNA gene using the primers 338F

356 (5'-ACTCCTACGGGAGGCAGCA-3') and 806R (5'-GGACTACHVGGGTWTCTAAT-3').

357 Two barcodes and six frameshifts were added to the 5' end of 338F and six frameshifts

358 were added to the $806 \mathrm{R}$ primers, based on the protocol by Lundberg et $\mathrm{al}^{40}$. Each PCR

359 reaction was performed in triplicate, and included a unique mixture of three frameshifted

360 primer combinations for each plate. PCR conditions were as follows: $5 \mu \mathrm{L}$ Kapa

361 Enhancer, $5 \mu \mathrm{L}$ Kapa Buffer A, $1.25 \mu \mathrm{L} 5 \mu \mathrm{M} 338 \mathrm{~F}, 1.25 \mu \mathrm{L} 5 \mu \mathrm{M}$ 806R, $0.375 \mu \mathrm{L}$ mixed

362 plant rRNA gene-blocking peptide nucleic acids (PNAs; 1:1 mix of $100 \mu \mathrm{M}$ plastid PNA 
363 and $100 \mu \mathrm{M}$ mitochondrial PNA ${ }^{40}$ ), $0.5 \mu \mathrm{L}$ Kapa dNTPs, $0.2 \mu \mathrm{L}$ Kapa Robust Taq, $8 \mu \mathrm{L}$

$364 \mathrm{dH}_{2} \mathrm{O}, 5 \mu \mathrm{L}$ DNA; temperature cycling: $95^{\circ} \mathrm{C}$ for $60 \mathrm{~s} ; 24$ cycles of $95^{\circ} \mathrm{C}$ for $15 \mathrm{~s} ; 78^{\circ} \mathrm{C}$

365 (PNA) for $10 \mathrm{~s} ; 50^{\circ} \mathrm{C}$ for $30 \mathrm{~s} ; 72^{\circ} \mathrm{C}$ for $30 \mathrm{~s} ; 4^{\circ} \mathrm{C}$ until use. Following PCR cleanup, using

366 AMPure beads (Beckman Coulter), the PCR product was indexed using 96 indexed 806R

367 primers with the Kapa HiFi Hotstart readymix with the same primers as above;

368 temperature cycling: $95^{\circ} \mathrm{C}$ for $60 \mathrm{~s} ; 9$ cycles of $95^{\circ} \mathrm{C}$ for $15 \mathrm{~s} ; 78^{\circ} \mathrm{C}(\mathrm{PNA})$ for $10 \mathrm{~s} ; 60^{\circ} \mathrm{C}$

369 for $30 \mathrm{~s} ; 72^{\circ} \mathrm{C}$ for $35 \mathrm{~s} ; 4^{\circ} \mathrm{C}$ until use. PCR products were purified using AMPure XP

370 magnetic beads (Beckman Coulter) and quantified with a Qubit 2.0 fluorometer

371 (Invitrogen). Amplicons were pooled in equal amounts and then diluted to $10 \mathrm{pM}$ for

372 sequencing. Sequencing was performed on an Illumina MiSeq instrument using a 600-

373 cycle V3 chemistry kit. DNA sequence data for this experiment is available at the NCBI

374 bioproject repository (accession PRJNA543313). The abundance matrix, metadata and

375 taxonomy are available at https://github.com/isaisg/variovoraxRGI.

e. $16 \mathrm{~S}$ amplicon sequence data processing

378 SynCom sequencing data were processed with MT-Toolbox ${ }^{41}$. Usable read output from

379 MT-Toolbox (that is, reads with $100 \%$ correct primer and primer sequences that 380 successfully merged with their pair) were quality filtered using Sickle ${ }^{42}$ by not allowing

381 any window with Q-score under 20. The resulting sequences were globally aligned to a 382 reference set of 16S rDNA sequences extracted from genome assemblies of SynCom 383 members. For strains that did not have an intact $16 \mathrm{~S}$ rDNA sequence in their assembly, 384 we sequenced the 16S rRNA gene using Sanger sequencing. The reference database 385 also included sequences from known bacterial contaminants and Arabidopsis organellar 
sequences. Sequence alignment was performed with USEARCH v7.109043 with the option 'usearch_global' at a 98\% identity threshold. On average, $85 \%$ of sequences matched an expected isolate. Our 185 isolates could not all be distinguished from each other based on the V3-V4 sequence and were thus classified into 97 unique sequences (USeqs). A USeq encompasses a set of identical (clustered at 100\%) V3-V4 sequences coming from a single or multiple isolates.

Sequence mapping results were used to produce an isolate abundance table. The remaining unmapped sequences were clustered into Operational Taxonomic Units (OTUs) using UPARSE44 implemented with USEARCH v7.1090, at 97\% identity. Representative OTU sequences were taxonomically annotated with the RDP classifier ${ }^{45}$ trained on the Greengenes database ${ }^{46}$ (4 February 2011). Matches to Arabidopsis organelles were discarded. The vast majority of the remaining unassigned OTUs belonged to the same families as isolates in the SynCom. We combined the assigned USeq and unassigned OTU count tables into a single count table. In addition to the raw count table, we created rarefied (1000 reads per sample) and relative abundance versions of the abundance matrix for further analyses.

The resulting abundance tables were processed and analyzed with functions from the ohchibi package (https://github.com/isaisg/ohchibi). An alpha diversity metric (Shannon diversity) was calculated using the diversity function from the vegan package v2.5-347. We used ANOVA to test for differences in alpha diversity between groups. Beta diversity analyses (Principal coordinate analysis, and canonical analysis of principal coordinates) 
were based on Bray-Curtis dissimilarity calculated from the relative abundance matrices.

We used the capscale function from the vegan R package v.2.5-347 to compute the canonical analysis of principal coordinates (CAP). To analyze the full dataset (all fraction, all abiotic treatments), we constrained by fraction and abiotic treatment while conditioning for the replica and experiment effect. We explored the abiotic conditions effect inside each of the four abiotic gradients tested (phosphate, salinity, $\mathrm{pH}$ and temperature). We performed the Fraction:abiotic interaction analysis within each fraction independently, constraining for the abiotic conditions while conditioning for the replica effect. In addition to CAP, we performed Permutational Multivariate Analysis of Variance (PERMANOVA) using the adonis function from the vegan package v2.5-347. We used the package DESeq2 v1.22.14 to compute the enrichment profiles for USeqs present in the count table.

We estimated the fraction effect across all the abiotic conditions tested by creating a group variable that merged the fraction variable and the abiotic condition variable together (e.g Root_OPi, Agar_OPi). We fitted the following model specification using this group variable:

Abundance $\sim$ Rep + Experiment + group

From the fitted model, we extracted, for all levels within the group variables, the following comparisons: Agar vs Root and Agar vs Shoot. A USeq was considered statistically significant if it had a false discovery rate (FDR) adjusted $p$-value $<0.05$. 
432 analyses are deposited in the following github repository:

433 https://github.com/isaisg/variovoraxRGI

434

435

f. Co-occurrence analysis

436 The relative abundance matrix (USeqs X Samples) was standardized across the USeqs

437 by dividing the abundance of each USeq in its sample over the mean abundance of that

438 USeq across all samples. Subsequently, we created a dissimilarity matrix based on the

439 Pearson correlation coefficient between all the pairs of strains in the transformed

440 abundance matrix, using the cor function in the stats base package in R. Finally,

441 hierarchichal clustering (method ward.D2, function hclust) was applied over the

442 dissimilarity matrix constructed above.

444 g. Heatmap and family enrichment analysis

445 We visualized the results of the GLM model testing the fraction effects across each

446 specific abiotic condition tested using a heatmap. The rows in the heatmap were ordered

447 according to the dendrogram order obtained from the USeqs co-occurrence analysis.

448 The heatmap was colored based on the $\log _{2}$ FoldChange output by the GLM model. We

449 highlighted in a black shade the comparisons that were significant $(q$-value $<0.05)$.

450 Finally, for each of the four modules we computed for each family present in that module

451 a hypergeometric test testing if that family was overrepresented (enriched) in that

452 particular module. Families whose FDR $p$-value $<0.1$ were visualized in the figure.

453

454 2. Deconstructing the SynCom to four modules of co-occurring strains (Fig. 2a, 2c and 
455 data S3).

456 a. Bacterial culture and plant-inoculation

457 Strains belonging to each module: A, B, C and D (Materials and Methods 1f) were grown

458 in separate deep 96-well plates and mixed as described above (Materials and Methods

459 1a). The concentration of each module was adjusted to $\mathrm{OD}_{600}=0.05(1 / 4$ of the

460 concentration of the full SynCom). Each module was spread on the plates either

461 separately, or in combination with another module at a total volume of $100 \mu \mathrm{L}$. In

462 addition, we included a full SynCom control and an uninoculated control, bringing the

463 number of SynCom combinations to 12 . We performed the experiment in two

464 independent replicates and each replicate included five plates per SynCom combination.

466 b. In vitro plant growth conditions

467 Seed sterilization and germination conditions were the same as Materials and Methods

468 1b. Plants were transferred to each of the SynCom-inoculated agar plates containing JM

469 without sucrose. Plates were placed in randomized order in growth chambers and grown

470 under a $16-\mathrm{h}$ dark/8-h light regime at $21^{\circ} \mathrm{C}$ day/18 ${ }^{\circ} \mathrm{C}$ night for 12 days. Upon harvest,

471 root morphology was measured.

472

473 c. Root and shoot image analysis

474 Plates were imaged twelve days post-transferring, using a document scanner. Primary

475 root length elongation was measured using Image $J^{49}$ and shoot area and total root

476 network were measured with WinRhizo software (Regent Instruments Inc.). 
d. Primary root elongation analyses

479 Primary root elongation was compared across the No Bacteria, full SynCom, single

480 modules and pairs of modules treatments jointly using an ANOVA model controlling for

481 the replicate effect. Differences between treatments were indicated using the confidence

482 letter display (CLD) derived from the Tukey's post hoc test implemented in the package

483 emmeans ${ }^{50}$.

484

3. Inoculating plants with all SynCom isolates separately (Fig. 2B, fig. S4 and data S4)

a. Bacterial culture and plant-inoculation.

487 Cultures from each strain in the SynCom were grown in KB medium and washed separately (Methods 1a), and $\mathrm{OD}_{600}$ was adjusted to 0.01 before spreading $100 \mu \mathrm{L}$ on plates. We performed the experiment in two independent replicates and each replicate included one plate per each of the 185 strains. In vitro growth conditions were the same

491 as in Materials and Methods 2b. Upon harvest, root morphology was measured

492 (Materials and Methods 2c). Isolates generating an average main root elongation of $<3$ $493 \mathrm{~cm}$ were classified as RGl-inducing strains.

a. Experimental design

497 To identify strains that revert RGI (Fig. 2D and data S5), we selected all 18 non-RGI 498 inducing strains in module A and co-inoculated them with each of four RGI inducing 499 strains, one from each module. The experiment also included uninoculated controls and controls consisting of each of the 22 strains inoculated alone, amounting to 95 separate 
501 bacterial combinations.

502

503 To confirm the ability of Variovorax and Burkholderia to attenuate RGI induced by diverse

504 bacteria (Fig. 2E and data S6), three RGI attenuating strains were co-inoculated with a

505 selection of $18 \mathrm{RGI}$ inducing strains. The experiment also included uninoculated controls

506 and controls consisting of each of the 21 strains inoculated alone. Thus, the experiment

507 consisted of 76 separate bacterial combinations. We performed each of these two

508 experiments in two independent replicates and each replicate included one plate per

509 each of the strain combinations.

510

511 b. Bacterial culture and plant-inoculation

512 All strains were streaked on agar plates, then transferred to $4 \mathrm{ml}$ liquid $\mathrm{KB}$ medium for

513 over-night growth. Cultures were then washed, and $\mathrm{OD}_{600}$ was adjusted to 0.02 before

514 mixing and spreading $100 \mu \mathrm{L}$ on each plate. Upon harvest, root morphology was

515 measured (Materials and Methods 2c) and plant RNA was harvested and processed from

516 uninoculated samples, and from samples with Variovorax CL14, Arthrobacter CL28 and

517 the combination of both (Materials and Methods 4d).

c. Primary root elongation analysis.

520 We fitted ANOVA models for each RGI-inducing strain tested. Each model compared the

521 primary root elongation with the RGI inducing strains alone against root elongation when

522 the RGI inducing strain was co-inoculated with other isolates. The $p$-values for all the

523 comparisons were corrected for multiple testing using false discovery rate (FDR). 
d. RNA extraction

526

RNA was extracted from $A$. thaliana seedlings following Logemann et a ${ }^{51}$. Four seedlings were harvested from each sample and samples were flash frozen and stored at $-80{ }^{\circ} \mathrm{C}$ until processing. Frozen seedlings were ground using a TissueLyzer II (Qiagen), then homogenized in a buffer containing $400 \mu \mathrm{L}$ of Z6-buffer; $8 \mathrm{M}$ guanidine $\mathrm{HCl}, 20$ mM MES, $20 \mathrm{mM}$ EDTA at pH 7.0. $400 \mu \mathrm{L}$ phenol:chloroform:isoamylalcohol, 25:24:1 was added, and samples were vortexed and centrifuged (20,000 g, 10 minutes) for phase separation.

532 The aqueous phase was transferred to a new $1.5 \mathrm{~mL}$ Eppendorf tube and 0.05 volumes of $1 \mathrm{~N}$ acetic acid and 0.7 volumes $96 \%$ ethanol were added. The RNA was precipitated at $-20^{\circ} \mathrm{C}$ overnight. Following centrifugation $\left(20,000 \mathrm{~g}, 10\right.$ minutes, $\left.4^{\circ} \mathrm{C}\right)$, the pellet was washed with $200 \mu \mathrm{L}$ sodium acetate (pH 5.2) and 70\% ethanol. The RNA was dried and dissolved in $30 \mu \mathrm{L}$ of ultrapure water and stored at $-80^{\circ} \mathrm{C}$ until use.

539 Illumina-based mRNA-Seq libraries were prepared from $1 \mu \mathrm{g}$ RNA following ${ }^{4}$. mRNA was 540 purified from total RNA using Sera-mag oligo(dT) magnetic beads (GE Healthcare Life 541 Sciences) and then fragmented in the presence of divalent cations $\left(\mathrm{Mg}^{2+}\right)$ at $94^{\circ} \mathrm{C}$ for 6 542 minutes. The resulting fragmented mRNA was used for first-strand cDNA synthesis using 543 random hexamers and reverse transcriptase, followed by second-strand cDNA synthesis 544 using DNA Polymerase I and RNAseH. Double-stranded cDNA was end-repaired using 545 T4 DNA polymerase, T4 polynucleotide kinase, and Klenow polymerase. The DNA 546 fragments were then adenylated using Klenow exo-polymerase to allow the ligation of 
547 Illumina Truseq HT adapters (D501-D508 and D701-D712). All enzymes were purchased

548 from Enzymatics. Following library preparation, quality control and quantification were

549 performed using a 2100 Bioanalyzer instrument (Agilent) and the Quant-iT PicoGreen

550 dsDNA Reagent (Invitrogen), respectively. Libraries were sequenced using Illumina

551 HiSeq4000 sequencers to generate 50-bp single-end reads.

552

f. RNA-Seq read processing

554 Initial quality assessment of the Illumina RNA-Seq reads was performed using FastQC

$555 \mathrm{v} 0.11 .7^{52}$. Trimmomatic $\mathrm{v} 0.36^{53}$ was used to identify and discard reads containing the

556 Illumina adaptor sequence. The resulting high-quality reads were then mapped against

557 the TAIR10 Arabidopsis reference genome using HISAT2 v2.1.0 54 with default

558 parameters. The featureCounts function from the Subread package ${ }^{55}$ was then used to

559 count reads that mapped to each one of the 27,206 nuclear protein-coding genes.

560 Evaluation of the results of each step of the analysis was performed using MultiQC v1. $1^{56}$.

561 Raw sequencing data and read counts are available at the NCBI Gene Expression

562 Omnibus accession number GSE131158.

564 5. Variovorax drop-out experiment (Fig. 3A-C and data S7)

565 a. Bacterial culture and plant-inoculation.

566 The entire SynCom, excluding all 10 Variovorax isolates and all five Burkholderia isolates

567 was grown and prepared as described above (Materials and Methods 1a). The Variovorax

568 and Burkholderia isolates were grown in separate tubes, washed and added to the rest

569 of the SynCom to a final $\mathrm{OD}_{600}$ of 0.001 (the calculated $\mathrm{OD}_{600}$ of each individual strain in 
a 185-Member SynCom at a total of $\mathrm{OD}_{600}$ of 0.2 ), to form the following five mixtures: (i)

571 Full community: all Variovorax and Burkholderia isolates added to the SynCom; (ii)

572 Burkholderia drop-out: only Variovorax isolates added to the SynCom; (iii) Variovorax

573 drop-out: only Burkholderia isolates added to the SynCom; (iv) Variovorax and

574 Burkholderia drop-out: no isolates added to the SynCom; (v) Uninoculated plants: no

575 SynCom. The experiment consisted of six plates per SynCom mixture, amounting to 30

576 plates. Upon harvest, root morphology was measured and analyzed (Materials and

577 Methods 1c,4c); and Bacterial DNA (Materials and Methods 1d) and plant RNA (Materials

578 and Methods 4d-e) were harvested and processed.

579

6. Variovorax drop-out under varying abiotic contexts (Fig. 3E and data S7)

a. Bacterial culture and plant-inoculation.

582 The composition of JM in the agar plates was amended to produce abiotic environmental variation. These amendments included salt stress (150 mM NaCl), low Phosphate (10 $\mu \mathrm{M}$

Phosphate), high $\mathrm{pH}(\mathrm{pH} 8.2)$ and high temperature (plates incubated at $31^{\circ} \mathrm{C}$ ), as well as an un-amended JM control. Additionally, we tested a different media (1/2-strength Murashige and Skoog [MS]) and a soil-like substrate. As a soil-like substrate, we used calcined clay (Diamond Pro), prepared as follows: $100 \mathrm{~mL}$ of clay was placed in Magenta GA7 jars. The jars were then autoclaved twice. $40 \mathrm{~mL}$ of liquid JM was added to the

589 Magenta jars, with the corresponding bacterial mixture spiked into the media at a final

$590 \mathrm{OD}_{600}$ of $5 \mathrm{E}-4$. Four 1 -week old seedlings were transferred to each vessel, and vessels 591 were covered with Breath-Easy gas permeable sealing membrane (Research Products 592 International) to maintain sterility and gas exchange. 
594 The entire SynCom, excluding all 10 Variovorax isolates was grown and prepared as

595 described above (Materials and Methods 1a). The Variovorax isolates were grown in 596 separate tubes, washed and added to the rest of the SynCom to a final OD 600 of 0.001

597 (the calculated $\mathrm{OD}_{600}$ of each individual strain in a 185-Member SynCom at an $\mathrm{OD}_{600}$ of 598 0.2), to form the following five mixtures: (i) Full community: all Variovorax isolates added 599 to the SynCom; (ii) Variovorax drop-out: no isolates added to the SynCom; (iii) 600 Uninoculated plants: no SynCom.

601

602 We inoculated all three SynCom combinations in all seven abiotic treatments, amounting 603 to 21 experimental conditions. We performed the experiment in two independent 604 replicates and each replicate included three plates per experimental conditions, 605 amounting to 63 plates per replicate. Upon harvest, root morphology was measured 606 (Materials and Methods 2c); and Bacterial DNA (Materials and Methods 1c-e) and plant 607 RNA (Materials and Methods 4d-f) were harvested and processed.

608

b. Root image analysis

610 For agar plates, roots were imaged as described above (Materials and Methods 2c). For

611 calcined clay pots, four weeks post-transferring, pots were inverted, and whole root 612 systems were gently separated from the clay by washing with water. Root systems were 613 spread over an empty petri dish and scanned using a document scanner. 
616 Primary root elongation was compared between SynCom treatments within each of the

617 different abiotic contexts tested independently. Differences between treatments were

618 indicated using the confidence letter display (CLD) derived from the Tukey's post hoc

619 test implemented in the package emmeans.

620

621

d. Bacterial $16 \mathrm{~S}$ data analysis

622 To be able to compare shifts in the community composition of samples treated with and 623 without the Variovorax genus, we in silico removed the 10 Variovorax isolates from the

624 count table of samples inoculated with the Full community treatment. We then merged 625 this count table with the count table constructed from samples inoculated without the 626 Variovorax genus (Variovorax drop-out treatment). Then, we calculated a relative 627 abundance of each USeq across all the samples using the merged count matrix. Finally, 628 we applied Canonical Analysis of Principal Coordinates (CAP) over the merged relative 629 abundance matrix to control for the replica effect. In addition, we utilized the function adonis from the vegan $\mathrm{R}$ package to compute a PERMANOVA test over the merged relative abundance matrix and we fitted a model evaluating the fraction and SynCom

632 (presence of Variovorax) effects over the assembly of the community.

a. Bacterial culture and plant-inoculation.

636 Strains belonging to modules A (excluding Variovorax), C and D were grown in separate 637 wells in deep 96-well plates and mixed as described above (Materials and Methods 1a). 638 The concentration of each module was adjusted to $\mathrm{OD}_{600}=0.05(1 / 4$ of the concentration 
639 of the full SynCom). The Variovorax isolates were grown in separate tubes, washed and 640 added to the rest of the SynCom to a final $\mathrm{OD}_{600}$ of 0.001.

641

642 In a separate experiment, the 35-member SynCom used by Castrillo et $\mathrm{al}^{2}$ was grown, 643 excluding Variovorax CL14, to create a taxonomically diverse, Variovorax-free subset of 644 the full 185 community. The concentration of this SynCom was adjusted to $\mathrm{OD}_{600}=0.05$.

645 The Variovorax isolates were grown in separate tubes, washed and added to the rest of 646 the SynCom to a final $\mathrm{OD}_{600}$ of 0.001.

647

648 These two experiments included the following mixtures (fig S7 and data S7): (i) Module 649 A excluding Variovorax; (ii) Module C; (iii) Module D; (iv) Module A including Variovorax; 650 (v) Module C + all 10 Variovorax; (vi) Module D + all 10 Variovorax; (vii) 35-member

651 SynCom excluding Variovorax; (viii) 34-member SynCom + all 10 Variovorax; (ix) 652 uninoculated control. The experiment with modules $A, C$ and $D$ was performed in two 653 independent experiments, with two plates per treatment in each. The experiment with 654 the 34-member SynCom was performed once, with 5 plates per treatment. Upon 655 harvest, root morphology was measured (Materials and Methods 2c). 656 b. Primary root elongation analysis.

658 We directly compared differences between the full SynCom and Variovorax drop-out 659 treatment using a $t$-test and adjusting the $p$-values for multiple testing using false 660 discovery rate. 
8. Phylogenetic inference of the SynCom and Variovorax isolates (Fig 2A, fig. S1A, S4, S7 and S9A-B)

664 To build the phylogenetic tree of the SynCom isolates, we used the super matrix 665 approach previously described $i^{24}$. We scanned 120 previously defined marker genes across the 185 isolate genomes from the SynCom utilizing the hmmsearch tool from the hmmer v3.1b257. Then, we selected 47 markers that were present as single copy genes in $100 \%$ of our isolates. Next, we aligned each individual marker using MAFFT ${ }^{58}$ and filtered low quality columns in the alignment using trimAl${ }^{59}$. Then, we concatenated all filtered alignments into a super alignment. Finally, FastTree v2.1 ${ }^{60}$ was used to infer the 671 phylogeny utilizing the WAG model of evolution. For the Variovorax relative's tree, we

672 chose 56 markers present as single copy across 124 Burkholderiales isolates and 673 implemented the same methodology described above.

a. Bacterial culture and plant-inoculation.

678 Fifteen Variovorax strains from across the genus' phylogeny were each co-inoculated with the RGI inducer Arthrobacter CL28. All 16 strains were grown in separate tubes, then washed, and $\mathrm{OD}_{600}$ was adjusted to 0.01 before mixing. Pairs of strains were mixed 681 in 1:1 ratios and spread at a total volume of $100 \mu \mathrm{L}$ onto agar prior to seedling transfer.

682 The experiment also included uninoculated controls and controls consisting of each of 683 the 16 strains inoculated alone. Thus, the experiment consisted of 32 separate bacterial 684 combinations. We performed the experiment one time, which included 3 plates per 
685 bacterial combination. Upon harvest, root morphology was measured (Materials and 686 Methods 2c). Primary root elongation was analyzed as described above (Materials and 687 Methods 4c).

688

10. Measuring root growth inhibition in tomato seedlings (fig. S10 and data S9)

a. Experimental design

691 This experiment included the following treatments: (i) No bacteria, (ii) Arthrobacter CL28,

(iii) Variovorax CL14 and (iv) Arthrobacter CL28 + Variovorax CL14. Each treatment was repeated in three separate agar plates with five tomato seedlings per plate. The experiment was repeated in two independent replicates.

All strains were grown in separate tubes, then washed, and $\mathrm{OD}_{600}$ was adjusted to 0.01 before mixing and spreading (Methods 3b). $400 \mu \mathrm{L}$ of each bacterial treatment was spread on $20 \times 20$ agar plates containing JM agar with no sucrose.

702 We used Heinz 1706 seeds. All seeds were soaked in sterile distilled water for 15 min, 703 then surface-sterilized with $70 \%$ bleach, $0.2 \%$ Tween-20 for $15 \mathrm{~min}$, and rinsed five 704 times with sterile distilled water to eliminate any seed-borne microbes on the seed 705 surface. Seeds were stratified at $4{ }^{\circ} \mathrm{C}$ in the dark for two days. Plants were germinated 706 on vertical square $10 \times 10 \mathrm{~cm}$ agar plates with JM containing $0.5 \%$ sucrose, for 7 days. 707 Then, 5 plants were transferred to each of the SynCom-inoculated agar plates. Upon 
708 harvest, root morphology was measured. (Materials and Methods 2c).

709

710

c. Primary root elongation analysis.

711 Differences between treatments were indicated using the confidence letter display (CLD)

712 derived from the Tukey's post hoc test from an ANOVA model.

11. Determination of Arthrobacter CL28 colony forming units from roots (fig. S11 and data S10)

Arabidopsis seedlings were inoculated with (i) Arthrobacter CL28 alone, (ii) Arthrobacter CL28 + Variovorax CL14 or (iii) Arthrobacter CL28 + Variovorax B4, as described above (Material and Methods 4b). Each bacterial treatment included four separate plates, with nine seedlings in each plate. Upon harvest, all seedlings were placed in pre-weighed 2 $\mathrm{mL}$ Eppendorf tubes containing three glass beads, three seedlings per tube (producing

722 beater (MP Biomedicals). The resulting suspension was serially diluted, then plated on

723 LB agar plates containing $50 \mu \mathrm{g} / \mathrm{mL}$ of Apramycin and colonies were counting after 724 incubation of 48 hours at $28^{\circ} \mathrm{C}$.

a. Detection of RGl-induced genes (Fig. 4A-B)

728 To measure the transcriptional response of the plant to the different SynCom 729 combinations, we used the R package DESeq2 v.1.22. $1^{48}$. The raw count genes matrixes 730 for the dropout and tripartite experiments were used independently to define 
731 differentially expressed genes (DEGs). For the analysis of both experiments we fitted the

732 following model specification:

733 Abundance Gene SynCom

734

735 From the fitted models we derived the following contrasts to obtain differentially expressed genes (DEGs). A gene was considered differentially expressed if it had a q-

737 value $<0.1$. For the tripartite system (Materials and Methods 4), we performed the

738 following contrasts: Arthrobacter CL28 vs No Bacteria (NB) and Arthrobacter CL28 vs

739 Arthrobacter CL28 co-inoculated with Variovorax CL14. The logic behind these two

740 contrasts was to identify genes that were induced in RGI plants (Arthrobacter CL28 vs

741 NB) AND repressed by Variovorax CL14. For the dropout system (Materials and Methods

742 5), we performed the following contrasts, Variovorax drop-out vs NB, and Variovorax

743 drop-out vs full SynCom. The logic behind these two contrasts was identical to the

744 tripartite system: to identify genes that are associated with the RGI phenotype

745 (Variovorax drop-out vs NB contrast) AND repressed when Variovorax are present 746 (Variovorax drop-out vs full SynCom contrast).

748 For visualization purposes, we applied a variance stabilizing transformation (DESeq2) to

749 the raw count gene matrix. We then standardized each gene expression (z-score) along

750 the samples. We subset DEGs from this standardized matrix and calculated the mean z-

751 score expression value for each SynCom treatment.

752

753 To identify the tissue specific expression profile of the 18 intersecting genes between 
754 the tripartite and dropout systems, we downloaded the spatial expression profile of each

755 gene from the Klepikova atlas ${ }^{27}$ using the Bio-analytic resource of plant biology platform.

756 Then, we constructed a spatial expression matrix of the 18 genes and computed

757 pairwise Pearson correlation between all pairs of genes. Finally, we applied hierarchical

758 clustering to this correlation matrix.

759

760

b. Comparison with acute auxin response dataset (Figure 4C)

761 We applied the variance stabilizing transformation (DESeq2) to the raw count gene

762 matrix. We then standardized each gene expression (z-score) along the samples. From

763 this matrix, we subsetted 12 genes that in a previous study ${ }^{28}$ exhibited the highest fold

764 change between auxin treated and untreated samples. Finally, we calculated the mean

$765 \mathrm{z}$-score expression value of each of these 12 genes across the SynCom treatments. We

766 estimated the statistical significance of the trend of these 12 genes between a pair of

767 SynCom treatments (Full SynCom vs Variovorax drop-out, Arthrobacter CL28 vs

768 Arthrobacter CL28 plus Variovorax CL14) using a permutation approach: we estimated

769 a $p$-value by randomly selecting 12 genes 10000 times from the expression matrix and

770 comparing the mean expression between the two SynCom treatments (e.g Full SynCom

771 vs Variovorax drop-out) with the actual mean expression value from the 12 genes

772 reported as robust auxin markers.

773

774 13. Measuring the ability of Variovorax to attenuate RGI induced by small molecules

775 (Figure 4D and data S13) indole-3-acetic acid (IAA), 2,4-Dichlorophenoxyacetic acid (2,4-

776 D), ethylene (the ethylene precursor 1-Aminocyclopropane-1-carboxylic acid [ACC]), 
777 cytokinins (Zeatin, 6-Benzylaminopurine) and flagellin 22 peptide (flg22) (Fig. 4d).

778

a. Bacterial culture and plant-inoculation.

779 We embedded each of the following compounds in JM plates: $100 \mathrm{nM}$ Indole acetic acid

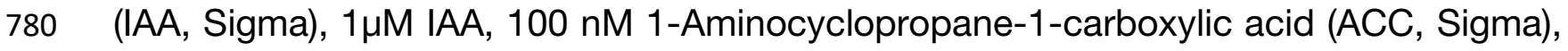
781100 nM 2,4-Dichlorophenoxyacetic acid (2,4-d, Sigma), 100 nM flagellin 22 (flg22, 782 PhytoTech labs), 100 nM 6-Benzylaminopurine (BAP, Sigma) and 100 nM Zeatin (Sigma).

783 Plates with each compound were inoculate with one of the Variovorax strains CL14, 784 MF160, B4 or YR216 or with Burkholderia CL11. These strains were grown in separate 785 tubes, then washed, and $\mathrm{OD}_{600}$ was adjusted to 0.01 before spreading $100 \mu \mathrm{L}$ on plates. 786 In addition, we included uninoculated controls for each compound. We also included unamended JM plates inoculated with the RGI inducer Arthrobacter CL28 co-inoculated with each of the Variovorax/Burkholderia strains or alone. Thus, the experiment included 42 individual treatments. The experiment was repeated twice, with three independent replicates per experiment. Upon harvest, root morphology was measured (Materials and Methods 2c).

b. Primary root elongation analysis.

794 Primary root elongation was compared between bacterial treatments within each of root growth inhibition treatments tested. Differences between treatments were estimated as 796 described above (Materials and Methods 4c). We plotted the estimated means with 95\% 797 confidence interval of each bacterial treatment across the different RGI treatments. 
800

801

802

803

804

805

806

807

808

809

810

811

812

813

814

815

816

817

818

819

820

821

822 GFP fluorescence in the root elongation zone of 8-10 plants per treatment were

Variovorax $\mathrm{CL} 14$ was grown in $5 \mathrm{~mL}$ cultures for 40 hours at $28^{\circ} \mathrm{C}$ in $1 \times \mathrm{M} 9$ minimal salts media (Sigma M6030) supplemented with $2 \mathrm{mM} \mathrm{MgSO}_{4}, 0.1 \mathrm{mM} \mathrm{CaCl}_{2}, 10 \mu \mathrm{M} \mathrm{FeSO}$, and a carbon source: either $15 \mathrm{mM}$ succinate alone, $0.4 \mathrm{mM}$ Indole-3-acetic acid (IAA) with $0.5 \%$ Ethanol for IAA solubilization, or both. Optical density at $600 \mathrm{~nm}$ and IAA concentrations were measured at six time points. IAA concentrations were measured using the Salkowski method modified from ${ }^{61} .100 \mu \mathrm{L}$ of Salkowski reagent $\left(10 \mathrm{mM} \mathrm{FeCl}_{3}\right.$ in $35 \%$ perchloric acid) was mixed with $50 \mu \mathrm{L}$ culture supernatant or IAA standards and color was allowed to develop for 30 min prior to measuring the absorbance at 530nm.

15. Measuring plant Auxin response in-vivo using a bioreporter line (Fig. 4E-F and data S14)

a. Bacterial culture and plant-inoculation.

7-day old transgenic Arabidopsis seedlings expressing the DR5::GFP reporter construct ${ }^{62}$ were transferred onto plates containing: (i) 100 nM IAA, (ii) Arthrobacter CL28, (iii) 100 IAA + Variovorax CL14, (iv) Arthrobacter CL28 + Variovorax CL14, (v) the Variovorax drop-out SynCom, (vi) the full SynCom, (vii) uninoculated plates. For treatments ii, iii, Bacterial strains were grown in separate tubes, then washed, and $\mathrm{OD}_{600}$ was adjusted to 0.01. For treatment iv, OD-adjusted cultures were mixed in 1:1 ratio and spread onto agar prior to seedling transfer. Cultures for treatments $v$ and vi were prepared as described above (Materials and Methods 6a).

b. Fluorescence microscopy. 
823 visualized using a Nikon Eclipse 80i fluorescence microscope at days 1, 3, 6, 9 and 13

824 post inoculation. The experiment was performed in two independent replicates.

825

826 From each root imaged, 10 random $30 \times 30$ pixel squares were sampled and average

827 GFP intensity was measured using image ${ }^{49}$. Treatments were compared within each

828 time point using ANOVA tests with Tukey's post hoc in the R base package emmeans.

829 For visualization purposes we plotted the estimated means of each bacterial across the

830 different timepoints.

831

832 16. Measuring the dual role of auxin and ethylene perception in SynCom-induced RGI

833 (Fig. 4F and data S15)

834

a. Bacterial culture and plant-inoculation.

835 We transferred four 7-day old wild type seedling and four axr1-2 seedlings to each plate

836 in this experiment. The plates contained one of five bacterial treatments: (i) Arthrobacter

837 CL28, (ii) Arthrobacter CL28 + Variovorax CL14, (iii) Variovorax drop-out SynCom, (iv) Full

838 SynCom, (v) uninoculated, prepared as described above (Materials and Methods 15a)

839 Plates were placed vertically inside sealed 86 X $68 \mathrm{~cm}$ Ziploc bags. In one of the bags, 840 we placed an open water container with 802.5 gram sachets containing $0.014 \%$ 1-MCP

841 (Ethylene Buster, Chrystal International BV). In the second bag we added, as a control, 842 an open water container. Both bags were placed in the growth chamber for 12 days. 843 After 6 days of growth, we added 32 additional sachets to the 1-MCP-treated bag to 844 maintain 1-MCP concentrations in the air. Upon harvest, root morphology was measured 845 (Materials and Methods 2c). 
b. Primary root elongation analysis.

848 Primary root elongation was standardized to the No bacteria control of each genotype, 849 and compared between genotype/1-MCP treatments within the Arthrobacter CL28

850 treatment and the Variovorax drop-out SynCom treatment, independently. Differences 851 between treatments were estimated as described above (Materials and Methods 4c). We

852 plotted the estimated means with $95 \% \mathrm{Cl}$ of each bacterial treatment across the four 853 genotypes. We calculated the interquartile range for the Full and Arthrobacter 854 CL28/Variovorax CL14 treatments pooling the four genotypes/treatments.

17. Preparation of binarized plant images (Fig 2C, 3B and fig. S5-S6)

857 To present representative root images, we increased contrast and subtracted 858 background in imageJ, then cropped the image to select representative roots. 859 Neighboring roots were manually erased from the cropped image.

861 18. Mining Variovorax genomes for auxin degradation operons and ACC-deaminase 862 genes.

863 We used local alignment (BLASTp) to search for the presence of the 10 genes 864 (iacABCDEFGHIY) from a previously characterized auxin degradation operon in a 865 different genus ${ }^{18}$ across the 10 Variovorax isolates in our SynCom. A minimal set of 7 of 866 these genes (iacABCDEFI) was shown to be necessary and sufficient for auxin 867 degradation ${ }^{18}$. We identified homologs for these genes across the Variovorax phylogeny 868 (Extended Figure 5a) at relatively low sequence identity (27-48\%). Two genes of the 
869 minimal set of 7 genes did not have any homologs in most Variovorax genomes (iacB

870 and $i a c l)$. In addition to the iac operon, we scanned the genomes for the auxin

871 degradation operon described by Ebenau-Jehle et al ${ }^{63}$ and could not identify it in any of

872 the Variovorax isolates.

873

874 We also searched for the ACC deaminase gene by looking for the KEGG orthology id

875 K01505 (1-aminocyclopropane-1-carboxylate deaminase) across the IMG annotations 876 available for all our genomes.

877

878 19. Variovorax CL14 RNA-Seq in monoculture and in co-culture with Arthrobacter CL28

879 a. Bacterial culture

880 Variovorax CL14 was grown either alone or in co-culture with Arthrobacter CL28 in 5mL

881 of 1/10 2xYT medium (1.6 g/L tryptone, $1 \mathrm{~g} / \mathrm{L}$ yeast extract, $0.5 \mathrm{~g} / \mathrm{L} \mathrm{NaCl})$ in triplicate.

882 The mono-culture was inoculated at $\mathrm{OD}_{600}$ of 0.02 and the co-culture was inoculated

883 with $\mathrm{OD}_{600}$ of 0.01 of each strain. Cultures were grown at $28^{\circ} \mathrm{C}$ to early stationary phase

884 (approximately 22 hours) and cells were harvested by centrifugation at $4100 \times \mathrm{g}$ for 15

885 min and frozen at $-80^{\circ} \mathrm{C}$ prior to RNA extraction.

886

887

b. RNA extraction and RNA-Seq

888 Cells were lysed for RNA extraction using TRIzol Reagent (Invitrogen) according to the 889 manufacturer instructions. Following cell lysis and phase separation, RNA was purified 890 using the RNeasy Mini kit (Qiagen) including the optional on column DNase Digestion 891 with the RNAse-Free DNase Set (Qiagen). Total RNA quality was confirmed on the 2100 
892 Bioanalyzer instrument (Agilent) and quantified using a Qubit 2.0 fluorometer (Invitrogen).

893 RNA-Seq libraries were prepared using the Universal Prokaryotic RNA-Seq, Prokaryotic

894 AnyDeplete kit (Tecan, formerly NuGEN). Libraries were pooled and sequenced on the

895 Illumina HiSeq4000 platform to generate 50-bp single-end reads.

896

897

c. RNA-Seq analysis

898

We mapped the generated raw reads to the Variovorax CL14 genome (fasta file available on github.com/isaisg/variovoraxRGI) using bowtie2 with the 'very-sensitive' flag. We then counted hits to each individual coding sequence (CDS) annotated for the Variovorax the Variovorax CL14 gff file (available on github.com/isaisg/variovoraxRGI) and using the default parameters with the flag allowMultiOverlap=FALSE. Finally, we used DESeq2 to estimate differentially expressed genes (DEGs) between treatments with the corresponding fold change estimates and false discovery adjusted p-values.

909 High molecular weight Variovorax CL14 genomic DNA was isolated by phenol-

911 England Biolabs), and separated on the BluePippin (Sage Science) to isolate DNA

912 fragments $>12.5 \mathrm{~kb}$. Vector backbone was prepared by amplifying pBBR-1MCS2 ${ }^{64}$ 913 using Phusion polymerase (New England Biolabs) with primers JMC277-JMC278 914 (Supplementary Table 19), digesting the PCR product with BamHI-HF (New England 
915 Biolabs), dephosphorylating with Quick CIP (New England Biolabs), and gel extracting

916 using the QIAquick Gel Extraction Kit (Qiagen). The prepared Variovorax CL14 genomic

917 DNA fragments were ligated to the prepared pBBR1-MCS2 vector backbone using

918 ElectroLigase (New England Biolabs) and transformed by electroporation into NEB 10-

919 beta Electrocompetent E. coli (New England Biolabs). Clones were selected by blue-

920 white screening on LB plates containing $1.5 \%$ agar, $50 \mu \mathrm{g} / \mathrm{mL}$ kanamycin, $40 \mu \mathrm{g} / \mathrm{mL}$ X-

921 gal (5-bromo-4-chloro-3-indolyl- $\beta$-D-galactopyranoside), and $1 \mathrm{mM}$ Isopropyl $\beta$ - d-1-

922 thiogalactopyranoside (IPTG) at $37^{\circ} \mathrm{C}$. White colonies were screened by colony PCR

923 using Taq polymerase and JMC247-JMC270 primers (Supplementary Table 19) to

924 eliminate clones with small inserts. The screened library clones were picked into LB

925 media $+50 \mu \mathrm{g} / \mathrm{mL}$ kanamycin, grown at $37^{\circ} \mathrm{C}$, and stored at $-80^{\circ} \mathrm{C}$ in $20 \%$ glycerol. The

926 Variovorax CL14 genomic library comprises approximately 3,500 clones with inserts

$927>12.5 \mathrm{~kb}$ in vector pBBR1-MCS2 in NEB 10-beta $E$. coli.

928

929

b. Library screening for IAA degradation

930 To screen the Variovorax CL14 genomic library for IAA degradation, the E. coli clones

931 were grown in LB media containing $50 \mu \mathrm{g} / \mathrm{mL}$ kanamycin, $1 \mathrm{mM}$ IPTG, $0.05 \mathrm{mg} / \mathrm{mL} \mathrm{IAA}$,

932 and $0.25 \%$ ethanol from IAA solubilization for 3 days at $37^{\circ} \mathrm{C}$. Salkowski reagent $(10 \mathrm{mM}$

$933 \mathrm{FeCl}_{3}$ in $35 \%$ perchloric acid) was mixed with culture supernatant 2:1 and color was

934 allowed to develop for 30 min prior to measuring the absorbance at 530nm. Two clones

935 from the library (plate 8 well E8 and plate2 well F10, henceforth Vector 1 and Vector 2,

936 respectively) were identified as degrading IAA. The Variovorax CL14 genes contained in

937 Vectors 1 and 2 were inferred by isolating plasmid from these clones using the 
ZymoPURE II Plasmid Midiprep Kit (Zymo Research) and Sanger sequencing the insert ends using primers JMC247 and JMC270 (Supplementary Table 19). Double digest of the purified plasmids with Sacl and EcoRV confirmed the size of the inserts. Vector 1 contains a $35 \mathrm{~kb}$ insert and Vector 2 contains a 15kb insert (nucleotide coordinates 29100-64406 and 52627-67679, respectively, from Variovorax CL14 scaffold Ga0102008_10005 (Fig. 5a). The genes in both inserts are in the same direction as the IPTG inducible Lac promoter used to drive LacZ-alpha expression for blue-white screening on pBBR1-MCS2.

21. Acidovorax Root219::EV and Acidovorax Root219::V2 construction and screening Triparental mating was used to mobilize Vector 2 or control empty vector (EV) pBBR1MCS2 from E. coli to Acidovorax Root219. Donor NEB 10-beta E. coli containing the vector for conjugation and helper strain E. coli pRK2013 ${ }^{65}$ were grown in LB medium containing $50 \mu \mathrm{g} / \mathrm{mL}$ kanamycin at $37^{\circ} \mathrm{C}$. Acidovorax Root219 was grown in $2 x Y T$ medium containing $100 \mu \mathrm{g} / \mathrm{mL}$ ampicillin at $28^{\circ} \mathrm{C}$. Bacteria were washed 3 times with 2xYT medium without antibiotics, mixed in a ratio of approximately 1:1:10 donor:helper:recipient, centrifuged and resuspended in 1/10 the volume and plated as a pool on LB agar plates without antibiotics and grown at $28^{\circ} \mathrm{C} .18-30$ hours later, exconjugantes were streaked on LB agar plates containing $50 \mu \mathrm{g} / \mathrm{mL}$ kanamycin and $100 \mu \mathrm{g} / \mathrm{mL}$ ampicillin to select only Acidovorax Root219 containing the conjugated vector. The resulting strains are designated Acidovorax Root219-EV containing empty vector pBBR1-MCS2 and Acidovorax Root219-V2 containing Vector 2 (Methods 20b). In vitro IAA degradation was performed as in Methods 14 using M9 media with carbon 
961 sources: $15 \mathrm{mM}$ succinate, $0.1 \mathrm{mg} / \mathrm{mL}$ indole-3-acetic acid (IAA), and 0.5\% Ethanol with

962 the addition of $50 \mu \mathrm{g} / \mathrm{mL}$ kanamycin and $1 \mathrm{mM}$ IPTG. Primary root elongation

963 measurement was performed as in Methods 2c on MS medium with 1mM IPTG and RGI

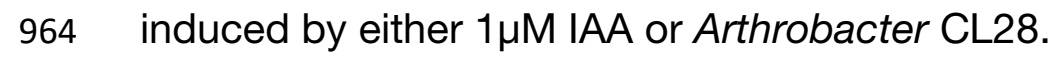

965

966

21. Variovorax hotspot 33 knockout construction and screening

967 The unmarked deletion mutant Variovorax CL14 $\Delta 2643613653-2643613677$ (Variovorax

968 CL14 $\mathrm{H}$ HS33) was constructed based on a genetic system developed for Burkholderia

969 spp. and its suicide vector pMo13066

970

971 a. Knockout suicide vector pJMC158 construction

972 The vector backbone was amplified from pMo130 using primers JMC203-JMC204

973 (Supplementary Table 19) with Q5 DNA polymerase (New England Biolabs), cleaned up

974 and treated with Dpnl (New England Biolabs). 1Kb regions for homologous

975 recombination flanking Variovorax CL14 genes 2643613653-2643613677 were amplified

976 using Q5 Polymerase (New England Biolabs) and primers JMC533-JMC534 and

977 JMC535-JMC536 (Supplementary Table 19). The vector was assembled with Gibson

978 Assembly Mastermix (New England Biolabs) at $50^{\circ} \mathrm{C}$ for 1 hour, transformed into NEB 5-

979 alpha chemically competent E. coli (New England Biolabs), and plated on LB agar with

$98050 \mu \mathrm{g} / \mathrm{mL}$ kanamycin. pJMC158 DNA was isolated from a clone using the ZR Plasmid

981 Miniprep Classic Kit (Zymo Research), sequence confirmed, and transformed into bi-

982 parental mating strain E. coli WM3064. E. coli strain WM3064 containing pJMC158 was

983 maintained on LB containing $50 \mu \mathrm{g} / \mathrm{mL}$ kanamycin and $0.3 \mathrm{mM}$ diaminopimelic acid (DAP) 
984 at $37^{\circ} \mathrm{C}$.

985 b. Conjugative transfer of pJMC158 into Variovorax CL14

986 For biparental mating, E. coli WM3064 was grown as above, and Variovorax CL14 was

987 grown in 2xYT medium containing $100 \mu \mathrm{g} / \mathrm{mL}$ ampicillin. Each strain was washed

988 separately 3 times with 2 xYT medium, then mixed at ratios between 1:1-1:10

989 donor:recipient, centrifuged and resuspended in approximately 1/10 the volume and

990 plated in a single pool on LB agar containing $0.3 \mathrm{mM}$ DAP and grown at $28^{\circ} \mathrm{C}$ overnight.

991 Exconjugants were streaked onto LB plates containing $100 \mu \mathrm{g} / \mathrm{mL}$ ampicillin $50 \mu \mathrm{g} / \mathrm{mL}$

992 kanamycin lacking DAP and grown at $28^{\circ} \mathrm{C}$ to select Variovorax $\mathrm{CL} 14$ strains that

993 incorporated suicide vector pJMC158. First crossover strains were subsequently purified

994 once by re-streaking and then individual colonies grown in LB with $100 \mu \mathrm{g} / \mathrm{mL}$ ampicillin

$99550 \mu \mathrm{g} / \mathrm{mL}$ kanamycin.

996

997 c. Resolution of pJMC158 integration and knockout strain purification and verification

998 To resolve the integration of pJMC158, first crossover strains were grown once in LB 999 medium containing $100 \mu \mathrm{g} / \mathrm{mL}$ ampicillin and $1 \mathrm{mM}$ IPTG then plated on media 1000 containing $10 \mathrm{~g} / \mathrm{L}$ tryptone, $5 \mathrm{~g} / \mathrm{L}$ yeast extract, $100 \mathrm{~g} / \mathrm{L}$ sucrose, $1.5 \%$ agar, $100 \mu \mathrm{g} / \mathrm{mL}$ 1001 ampicillin and $1 \mathrm{mM}$ IPTG. Colonies were picked into the same liquid media and grown

1002 once. The resulting strains were screened by PCR using Q5 polymerase for deletion of 1003 genes 2643613653-2643613677 using primers JMC568-JMC569 (Supplementary Table

1004 19). These strains were subsequently plate purified at least 3 times on LB $100 \mu \mathrm{g} / \mathrm{mL}$ 1005 ampicillin plates. To ensure strain purity, PCR primers were designed to amplify from 1006 outside into the genes that were deleted (primer pairs JMC571-JMC569 and JMC568- 
1007 JMC570, Supplementary Table 19). These PCR reactions were performed using Q5 1008 polymerase with wild type Variovorax CL14 as a control. All genomic DNA used for 1009 screening PCR was isolated using the Quick-DNA miniprep kit (Zymo Research). The 1010 resulting knockout strain was designated Variovorax CL14 $\triangle \mathrm{HS} 33$.

1011
d. Screening of Variovorax CL14 $\Delta \mathrm{HS} 33$

1013

In vitro IAA degradation was performed as in Methods 14 using M9 media with carbon

1014 sources: $15 \mathrm{mM}$ succinate, $0.1 \mathrm{mg} / \mathrm{mL}$ indole-3-acetic acid (IAA), and 0.5\% Ethanol.

1015 Primary root elongation measurement was performed as in Methods 2c on MS medium 1016 with 1mM IPTG and RGI induced by either $1 \mu \mathrm{M}$ IAA or Arthrobacter CL28.

\section{References}

1019 1. Hiruma, K. et al. Root Endophyte Colletotrichum tofieldiae Confers Plant Fitness

1020 Benefits that Are Phosphate Status Dependent. Cell 165, 464-474 (2016).

1021 2. Castrillo, G. et al. Root microbiota drive direct integration of phosphate stress 1022 and immunity. Nature 543, (2017).

1023 3. Durán, P. et al. Microbial Interkingdom Interactions in Roots Promote Arabidopsis Survival. Cell 175, 973-983.e14 (2018).

1025 4. Herrera Paredes, S. et al. Design of synthetic bacterial communities for 1026 predictable plant phenotypes. PLOS Biol. 16, e2003962 (2018).

1027 5. Huang, A. C. et al. A specialized metabolic network selectively modulates Arabidopsis root microbiota. Science 364, eaau6389 (2019). 
1029 6. Lundberg, D. S. et al. Defining the core Arabidopsis thaliana root microbiome.

1030

Nature 488, 86-90 (2012).

1031 7. Hogenhout, S. A., Van der Hoorn, R. A. L., Terauchi, R. \& Kamoun, S. Emerging

1032 Concepts in Effector Biology of Plant-Associated Organisms. Mol. Plant-Microbe

1033 Interact. 22, 115-122 (2009).

1034

8. Faure, D., Vereecke, D. \& Leveau, J. H. J. Molecular communication in the rhizosphere. Plant Soil 321, 279-303 (2009).

9. Herrera Paredes, S. et al. Design of synthetic bacterial communities for predictable plant phenotypes. PLoS Biol. 16, (2018).

10. Mylona, P., Pawlowski, K. \& Bisseling, T. Symbiotic Nitrogen Fixation. Plant Cell 7, 869-885 (1995).

11. Carlström, C. I. et al. Synthetic microbiota reveal priority effects and keystone strains in the Arabidopsis phyllosphere. Nat. Ecol. Evol. 3, 1445-1454 (2019).

12. Helman, Y. \& Chernin, L. Silencing the mob: disrupting quorum sensing as a means to fight plant disease. Mol. Plant Pathol. 16, 316-329 (2015).

13. Leveau, J. H. J. \& Lindow, S. E. Utilization of the plant hormone indole-3-acetic 1045 acid for growth by Pseudomonas putida strain 1290. Appl. Environ. Microbiol.

14. Zúñiga, A. et al. Quorum Sensing and Indole-3-Acetic Acid Degradation Play a 
(2013).

1051 15. Leadbetter, J. R. \& Greenberg, E. P. Metabolism of acyl-homoserine lactone quorum-sensing signals by Variovorax paradoxus. J. Bacteriol. 182, 6921-6 (2000).

16. Sun, S.-L. et al. The Plant Growth-Promoting Rhizobacterium Variovorax boronicumulans CGMCC 4969 Regulates the Level of Indole-3-Acetic Acid Synthesized from Indole-3-Acetonitrile. Appl. Environ. Microbiol. 84, e00298-18 (2018).

17. Gilbert, S. et al. Bacterial production of indole related compounds reveals their role in association between duckweeds and endophytes. Front. Chem. 6, (2018).

18. Donoso, R. et al. Biochemical and Genetic Bases of Indole-3-Acetic Acid (Auxin Phytohormone) Degradation by the Plant-Growth-Promoting Rhizobacterium Paraburkholderia phytofirmans PsJN. Appl. Environ. Microbiol. 83, e01991-16 (2017).

19. Leveau, J. H. J. \& Gerards, S. Discovery of a bacterial gene cluster for catabolism of the plant hormone indole 3-acetic acid. FEMS Microbiol. Ecol. 65, 238-250 (2008).

1067 20. Ludwig-Müller, J. Bacteria and fungi controlling plant growth by manipulating 1068 auxin: balance between development and defense. J. Plant Physiol. 172, 4-12 1069 (2015).

1070 21. Brumos, J. et al. Local Auxin Biosynthesis Is a Key Regulator of Plant 
Development. Dev. Cell 47, 306-318.e5 (2018).

1072 22. Edwards, J. A. et al. Compositional shifts in root-associated bacterial and

1073

1074

1075

1076

1077

1078

1079

1080

1081

1082

1083

1084

1085

1086

1087

1088

1089

1090

1091 archaeal microbiota track the plant life cycle in field-grown rice. PLOS Biol. 16, e2003862 (2018).

23. Fitzpatrick, C. R. et al. Assembly and ecological function of the root microbiome across angiosperm plant species. Proc. Natl. Acad. Sci. U. S. A. 115, E1157E1165 (2018).

24. Levy, A. et al. Genomic features of bacterial adaptation to plants. Nat. Genet. 50, $138-150$ (2018).

25. Kremer, J. M. et al. FlowPot axenic plant growth system for microbiota research. bioRxiv 254953 (2018). doi:10.1101/254953

26. Bai, Y. et al. Functional overlap of the Arabidopsis leaf and root microbiota. Nature 528, 364-369 (2015).

27. Klepikova, A. V., Kasianov, A. S., Gerasimov, E. S., Logacheva, M. D. \& Penin, A. A. A high resolution map of the Arabidopsis thaliana developmental transcriptome based on RNA-seq profiling. Plant J. 88, 1058-1070 (2016).

28. Uchida, N. et al. Chemical hijacking of auxin signaling with an engineered auxinTIR1 pair. Nat. Chem. Biol. 14, 299-305 (2018).

29. Takase, T. et al. $y d k 1-D$, an auxin-responsive $G H 3$ mutant that is involved in hypocotyl and root elongation. Plant J. 37, 471-483 (2004).

30. Chen, L., Dodd, I. C., Theobald, J. C., Belimov, A. A. \& Davies, W. J. The 
rhizobacterium Variovorax paradoxus 5C-2, containing ACC deaminase, promotes growth and development of Arabidopsis thaliana via an ethylenedependent pathway. J. Exp. Bot. 64, 1565-1573 (2013).

31. Cary, A. J., Liu, W. \& Howell, S. H. Cytokinin action is coupled to ethylene in its effects on the inhibition of root and hypocotyl elongation in Arabidopsis thaliana seedlings. Plant Physiol. 107, 1075-82 (1995).

32. Gómez-Gómez, L., Felix, G. \& Boller, T. A single locus determines sensitivity to bacterial flagellin in Arabidopsis thaliana. Plant J. 18, 277-284 (1999).

33. Nagpal, P. et al. AXR2 encodes a member of the AuX/IAA protein family. Plant Physiol. 123, 563-74 (2000).

34. Hall, A. E., Findell, J. L., Schaller, G. E., Sisler, E. C. \& Bleecker, A. B. Ethylene perception by the ERS1 protein in Arabidopsis. Plant Physiol. 123, 1449-58 (2000).

35. Gould, S. J. \& Vrba, E. S. Exaptation-a Missing Term in the Science of Form. Paleobiology 8, 4-15 (1982).

36. Bardoel, B. W. et al. Pseudomonas Evades Immune Recognition of Flagellin in Both Mammals and Plants. PLoS Pathog. 7, e1002206 (2011).

37. Carlström, C. I. et al. Synthetic microbiota reveal priority effects and keystone strains in the Arabidopsis phyllosphere. Nat. Ecol. Evol. 3, 1445-1454 (2019).

38. Thiergart, T. et al. Root microbiota assembly and adaptive differentiation among European Arabidopsis populations. bioRxiv 640623 (2019). doi:10.1101/640623 
1113 39. Finkel, O. M. et al. The effects of soil phosphorus content on plant microbiota are

1114 driven by the plant phosphate starvation response. PLOS Biol. 17, e3000534

1115 (2019).

1116 40. Lundberg, D. S., Yourstone, S., Mieczkowski, P., Jones, C. D. \& Dangl, J. L.

1117 Practical innovations for high-throughput amplicon sequencing. Nat. Methods

$1118 \quad 10,999-1002(2013)$.

1119 41. Yourstone, S. M., Lundberg, D. S., Dangl, J. L. \& Jones, C. D. MT-Toolbox:

1120 Improved amplicon sequencing using molecule tags. BMC Bioinformatics 15,

1121 (2014).

1122 42. Joshi, N. \& Fass, J. Sickle: A sliding-window, adaptive, quality-based trimming tool for FastQ files (Version 1.33) [Software]. Available at https://github.com/najoshi/sickle. (2011).

1125 43. Edgar, R. C. Search and clustering orders of magnitude faster than BLAST. 1126 Bioinformatics 26, 2460-2461 (2010).

1127 44. Edgar, R. C. UPARSE: highly accurate OTU sequences from microbial amplicon reads. Nat. Methods 10, 996-998 (2013).

1129 45. Wang, Q., Garrity, G. M., Tiedje, J. M. \& Cole, J. R. Naive Bayesian Classifier for Rapid Assignment of rRNA Sequences into the New Bacterial Taxonomy. Appl. Environ. Microbiol. 73, 5261-5267 (2007).

1132 46. DeSantis, T. Z. et al. Greengenes, a chimera-checked 16S rRNA gene database and workbench compatible with ARB. Appl. Environ. Microbiol. 72, 5069-72 
(2006).

47. Oksanen, J. et al. Package ‘vegan’. (2015).

1136

1137

1138

1139

1140

1141

1142

1143

1144

1145

1146

1147

1148

1149

1150

1151

1152

1153

1154

48. Love, M. I., Huber, W. \& Anders, S. Moderated estimation of fold change and dispersion for RNA-seq data with DESeq2. Genome Biol. 15, 550 (2014).

49. Schindelin, J. et al. Fiji: an open-source platform for biological-image analysis.

Nat. Methods 9, 676-682 (2012).

50. Package 'emmeans' Type Package Title Estimated Marginal Means, aka LeastSquares Means. (2019). doi:10.1080/00031305.1980.10483031

51. Logemann, J., Schell, J. \& Willmitzer, L. Improved method for the isolation of RNA from plant tissues. Anal. Biochem. 163, 16-20 (1987).

52. S, undefined A. Babraham Bioinformatics - FastQC A Quality Control tool for High Throughput Sequence Data. 3-5 (2018).

53. Bolger, A. M., Lohse, M. \& Usadel, B. Trimmomatic: a flexible trimmer for Illumina sequence data. Bioinformatics 30, 2114-20 (2014).

54. Kim, D., Langmead, B. \& Salzberg, S. L. HISAT: a fast spliced aligner with low memory requirements. Nat. Methods 12, 357-60 (2015).

55. Liao, Y., Smyth, G. K. \& Shi, W. The Subread aligner: fast, accurate and scalable read mapping by seed-and-vote. Nucleic Acids Res. 41, e108 (2013).

56. Ewels, P., Magnusson, M., Lundin, S. \& Käller, M. MultiQC: summarize analysis results for multiple tools and samples in a single report. Bioinformatics 32, 30473048 (2016). 
1155 57. Wheeler, T. J. \& Eddy, S. R. nhmmer: DNA homology search with profile HMMs. Bioinformatics 29, 2487-2489 (2013).

1157

58. Katoh, K. \& Standley, D. M. MAFFT multiple sequence alignment software version 7: improvements in performance and usability. Mol. Biol. Evol. 30, 77280 (2013).

59. Capella-Gutiérrez, S., Silla-Martínez, J. M. \& Gabaldón, T. trimAl: a tool for automated alignment trimming in large-scale phylogenetic analyses.

Bioinformatics 25, 1972-3 (2009).

1163

1164

1165

1166

1167

1168

1169

1170

1171

60. Price, M. N., Dehal, P. S. \& Arkin, A. P. FastTree 2 - Approximately MaximumLikelihood Trees for Large Alignments. PLoS One 5, e9490 (2010).

61. Gordon, S. A. \& Weber, R. P. COLORIMETRIC ESTIMATION OF INDOLEACETIC ACID. Plant Physiol. 26, 192-5 (1951).

62. Friml, J. et al. Efflux-dependent auxin gradients establish the apical-basal axis of Arabidopsis. Nature 426, 147-153 (2003).

63. Ebenau-Jehle, C. et al. Anaerobic metabolism of indoleacetate. J. Bacteriol. 194, 2894-2903 (2012).

64. Kovach, M. E. et al. Four new derivatives of the broad-host-range cloning vector pBBR1MCS, carrying different antibiotic-resistance cassettes. Gene 166, 175176 (1995).

65. Figurski, D. H. \& Helinski, D. R. Replication of an origin-containing derivative of plasmid RK2 dependent on a plasmid function provided in trans. Proc. Natl. 
Acad. Sci. U. S. A. 76, 1648-1652 (1979).

1177

1178

1179

1180

1181

1182

1183

1184

1185

1186

1187

1188

1189

1190

1191

1192

1193

1194

1195

1196

1197
66. Hamad, M. A., Zajdowicz, S. L., Holmes, R. K. \& Voskuil, M. I. An allelic exchange system for compliant genetic manipulation of the select agents Burkholderia pseudomallei and Burkholderia mallei. Gene 430, 123-131 (2009).

Acknowledgments: We thank Stratton Barth, Julia Shen, May Priegel, Dilan Chudasma, Darshana Panda, Izabella Castillo, Nicole Del Risco, and Chloe Lindberg for technical assistance; Dale Pelletier, DOE-ORNL and Paul Schulze-Lefert, MPIPZ, Koeln, Germany for strains; the Dangl lab microbiome group for useful discussions; Anna Stepanova, Jose Alonso, Javier Brumos (North Carolina State University, USA), Joseph Kieber, Jason Reed (UNC Chapel Hill), and Isaac Greenhut (University of California, Davis) for useful discussions and Derek Lundberg (Max Planck Institute for Developmental Biology, Tübingen, Germany), and Anthony Bishopp (University of Nottingham, UK) for critical comments on the manuscript. Funding: This work was supported by NSF INSPIRE grant IOS-1343020 and by Office of Science (BER), U.S. Department of Energy, Grant DESC0014395 to J.L.D. J.L.D is an Investigator of the Howard Hughes Medical Institute, supported by the HHMI. O.M.F was supported by NIH NRSA Fellowship F32-GM117758.

Author contributions: Conceptualization: O.M.F., I.S.G, G.C., J.L.D.; Methodology: O.M.F., I.S.G, G.C., T.F.L., J.M.C., P.J.P.L.T., E.D.W., C.R.F.; Software: I.S.G.; Validation: O.M.F., I.S.G, G.C., T.F.L., J.M.C.; Formal analysis: I.S.G, O.M.F., G.C.; Investigation: O.M.F., I.S.G, G.C., T.F.L., J.M.C., P.J.P.L.T., E.D.W., C.R.F; Resources: J.L.D.; Data Curation: I.S.G, O.M.F.; Writing - original draft: O.M.F, I.S.G, G.C., J.M.C.; 
1198 Writing - review and editing: T.F.L.,, C.D.J., J.L.D.; Visualization: I.S.G, O.M.F., G.C.;

1199 Supervision: J.L.D, C.D.J.; Project administration: J.L.D.; Funding acquisition: J.L.D.

1200 Competing interests: J.L.D. is a co-founder of, and shareholder in, AgBiome LLC, a 1201 corporation whose goal is to use plant-associated microbes to improve plant 1202 productivity

1203

1204 Data and materials availability:

1205 The 16S rRNA amplicon sequencing data associated with this study was deposited in 1206 the NCBI SRA archive under the project accession PRJNA543313. The raw 1207 transcriptomic data was deposited in the Gene Expression Omnibus (GEO) under the 1208 accession GSE131158. In addition to the supplementary tables, we deposited all scripts 1209 and additional data structures required to reproduce the results of this study in the 1210 following GitHub repository: https://github.com/isaisg/variovoraxRGI 